\begin{tabular}{|c|c|c|c|}
\hline & & $1-20$ & \\
Eiszeitalter $u$. Gegenwart & 49 & $10 \mathrm{Abb} ., 2 \mathrm{Tab}$. & Hannover 1999 \\
\hline
\end{tabular}

\title{
Holozäner Landschaftswandel im südlichen Oberrheintiefland und Schwarzwald
}

\author{
RÜDiger Mäckel \& ARne FriedmanN*) \\ - Holocene, Upper Rhine Valley and Black Forest, flood plains, loess valleys, Rhine, \\ geomorphodynamics, environmental changes, man-nature interactions -
}

\section{Inhaltsverzeichnis}

1 Problemstellung und Einführung in das Untersuchungsgebiet

2 Geomorphodynamische Aktivitätsphasen im Zartener Becken zur Zeit der Kelten und Römer

2.1 Aufbau und Altersstellung der Auenniveaus

2.2 Ursachen verstärkter Auensedimentation

3 Landschaftswandel in der Vorbergzone

3.1 Aufbau und Alter der Sedimente im Möhlintal

3.2 Einwirkung des Menschen auf die Landschaftsgenese innerhalb der Zeitscheibe III

4 Die Lößsohlentäler und Schwemmfächer im Kaiserstuhl und Tuniberg

4.1 Die Bohrprofile im Spührenlochtal bei Oberbergen

4.2 Der Sedimentaufbau der Sohlentäler bei Bickensohl und im Tuniberg

4.3 Die Schwemmfächer am Rande des Kaiserstuhls

4.4 Auswertung der Befunde in den Lößlandschaften von Kaiserstuhl und Tuniberg

5 Das Wasenweiler Ried und die Ostrheinproblematik

5.1 Pollenanalytische Untersuchungen

5.2 Landschaftswandel durch veränderte Geomorphodynamik

6 Schlußfolgerungen

7 Danksagung

8 Literaturverzeichnis

Kurzfassung: Die Untersuchungen zum holozänen Landschaftswandel im südlichen Oberrheintiefland und Schwarzwald konzentrieren sich auf die Zeitscheibe II ( 5500 bis 3000 v. Chr.) und III (1500 v. Chr. bis 500 n. Chr.) des DFG-Schwerpunktprogramms ,Wandel der Geo-Biosphäre während der letzten 15000 Jahre - Kontinentale Sedimente als Ausdruck sich ändernder Umweltbedingungen". Trotz einiger neolithischer Funde in den klimabegünstigten Lößgebieten wird der spürbare Eingriff des Menschen in die Naturlandschaft erst ab der Bronzezeit durch Kolluvien (Lößlehm) und Auensedimente nachgewiesen. Ein verstärkter Einfluß des Menschen auf die Abtragungsprozesse ist in der vorrömischen Eisenzeit, vor allem in der Latènezeit, zu

\footnotetext{
*) Anschrift der Verfasser: Prof. Dr. R. Mäckel und Dr. A. Friedmann, Institut für Physische Geographie der Albert-Ludwigs-Universität Freiburg, Werderring 4, D-79085 Freiburg i. Br.
}

beobachten. Die Ursachen dafür sind die Ausweitung der landwirtschaftlichen Nutzflächen durch die wachsende Bevölkerung bei gleichzeitiger Klimaverschlechterung. Eine einschneidende Auswirkung auf den Landschaftshaushalt hat bereits der Bergbau in der Latènezeit. Der anthropogene Einfluß auf die Geomorphodynamik verstärkt sich zur Römerzeit durch intensive landwirtschaftliche Nutzung, Siedlungen und den Bergbau. Mit dem Rückzug der Römer verringert sich die Siedlungsaktivität, und es tritt eine Stabilitätsphase mit Regeneration der Vegetation und Bodenbildung ein. Die alamannische Landnahme wirkte sich nur in den lößbedeckten und klimabegünstigten Siedlungsgebieten des Oberrheintieflandes und der Vorbergzone aus. Insgesamt führten die Aktivitäten der vorrömischen Eisenzeit und der Römerzeit zu den schwerwiegendsten anthropogenen Veränderungen im Untersuchungsgebiet während der drei Zeitscheiben.

\section{[Holocene environmental change in the south- ern Upper Rhine Valley and the Black Forest]}

\begin{abstract}
The investigations of the Holocene environmental changes in the southern Upper Rhine Valley and the Black Forest concentrate on the time slices II (5500$3000 \mathrm{BC})$ and III (1500 BC-500 AD) of the DFG (German Research Organization) research program: „Environmental changes of the Geo-Biosphere during the last 15000 years."
\end{abstract}

Despite of a few neolithic findings in the climatically favoured loess areas, a significant influence of man on the natural landscape is not recognized until the Bronze Age (colluvial and alluvial sediments). An increase of erosion caused by man is detectable from the pre-Roman Iron Age onwards, especially during the Latène. The causes are the expansion of farmland by the increasing population during a time of climatic deterioration. A strong influence on to the natural ecosystem can be shown for the mining activities during Latène times. The anthropogenic influence of the geomorphodynamics increases furthermore during the Roman period through intensive farming methods, settlement expansions and mining. With the retreat of the Romans the anthropogenic activities decline distinctively and a stable phase of vegetation regeneration and soil formation starts. The intrusion of the germanic tribe of the "Alamannen" into the area is only detectable in the loess covered and climatically favoured areas of the 
Upper Rhine Valley and the Black Forest foothills. Altogether the environmental changes and anthropogenic influences on to the landscape was most pronounced during the Iron Age and the Roman period in the investigated region.

\section{Problemstellung und Einführung in das Untersuchungsgebiet}

Die Untersuchungen zum holozänen Landschaftswandel wurden im Rahmen des DFGSchwerpunktprogramms „Wandel der Geo-Biosphäre während der letzten 15000 Jahre - Kontinentale Sedimente als Ausdruck sich verändernder Umweltbedingungen" durchgeführt, und zwar im regionalen Teilprojekt „Die paläoökologischen Umweltbedingungen im Oberrheintiefland und Schwarzwald im Neolithikum und zur Römerzeit - Fluviale Sedimente, Böden und Relief als Archive“ (Ma 557/11). Das DFG-Schwerpunktprogramm steht in Verbindung mit dem Internationalen Geosphären-Biosphärenprogramm (IGBP), hier speziell mit dem Kernprojekt „Past Global Changes" (PAGES). Ziel des Forschungsprogramms ist es, eine zuverlässige Kenntnis der Entwicklung der Geo-Biosphäre in der jüngsten erdgeschichtlichen Vergangenheit zu erhalten (ANDres et al. 1993, ANdres 1994). Diese Kenntnis wird als unabdingbare Voraussetzung für die Beurteilung des gegenwärtigen Zustands der GeoBiosphäre und ihrer zukünftigen Veränderungen angesehen. Die Veränderungen der Geo-Biosphäre werden aus natürlichen Archiven abgeleitet und zu archäologischen Befunden in Beziehung gesetzt. Damit soll erreicht werden, daß die natürlichen Veränderungen der Geo-Biosphäre von anthropogenen unterschieden werden. Entsprechend konzentrieren sich die Untersuchungen des Schwerpunktprogramms auf drei Zeitscheiben im Spät- und Postglazial mit unterschiedlicher anthropogener Einwirkung auf die Umwelt.

Als erste Zeitscheibe wurde der Übergang von der letzten Kaltzeit zum Holozän gewählt, und zwar insbesondere das Alleröd, also der Zeitabschnitt während und nach der Ablagerung der weit über Mitteleuropa verbreiteten und eindeutig zuordbaren Laacher-See-Tephra (LST) (SCHMINCKe \& BOGAARD 1991). In dieser Zeitscheibe war die Umwelt noch weitgehend von der menschlichen Einflußnahme unberührt (Übergang vom Jung- zum Spätpaläolithikum), und die natürlichen klimatischen Veränderungen wirkten einschneidend auf die paläoökologischen Rahmenbedingungen. Die zweite Zeitscheibe umfaßt das Atlantikum mit dem postglazialen Wärmeoptimum (5500 bis $3000 \mathrm{v}$. Chr.), wo der Mensch bereits spürbar in die Naturlandschaft einzugreifen begann („Neolithische Revolution“). Gerade in den lößbedeckten, klimatisch begünstigten Teilen des Oberrheintieflandes wurden deutliche Eingriffe des Menschen seit dem Übergang vom Mittel- zum Jungneolithikum nachgewiesen (Dehn 1988, Fingerlin 1991).

Zur dritten Zeitscheibe gehört das jüngere Subboreal und das ältere Subatlantikum, also der Zeitraum mit einsetzender intensiver Nutzung der natürlichen Ressourcen durch den Menschen (1500 v. Chr. bis 500 n. Chr.). Kulturgeschichtlich ist dieser Abschnitt besonders interessant für das Untersuchungsgebiet, da viele Funde für die Bronzezeit (1800 v. Chr. bis 800 v. Chr.), die vorrömische Eisenzeit (800 v. Chr. bis Christi Geburt), die Römerzeit (1. bis 4. Jahrhundert $n$. Chr.) und die frühe alamannische Landnahmezeit (5./6. Jh. n. Chr.) vorliegen.

Das Untersuchungsgebiet umfaßt den Mittleren und Südlichen Schwarzwald und das westlich vorgelagerte Oberrheintiefland, das naturräumlich in die randliche Vorbergzone und die Oberrheintiefebene untergliedert wird (Abb. 1). Forschungsobjekte bilden die fluvialen Sedimente der Talauen, die Hangsedimente in der Fußzone des Berglandes und die Moore sowohl in den Niederungen als auch auf den Hochflächen und in den Karen des Schwarzwaldes. Die Ablagerungen in den Talauen und auf den Hangfußflächen fallen überwiegend in die Zeitscheiben II und III des Schwerpunktprogramms. Die einzelnen naturräumlichen Einheiten (Bergland - Vorbergzone - Tiefland) weisen bezüglich Relief, Klima und Vegetation milieuspezifische Rahmenbedingungen auf. So spielen sich zur gleichen Zeit verschiedene Prozesse ab, die im hohen Bergland, z. B. in den Oberläufen der Entwässerungssysteme zur Erosion, in den mittleren und unteren Lagen zur Sedimentation in den Talauen führen. Gleiche Ursachen, wie z. B. ein Klimawechsel, bewirken ebenfalls verschiedenartige Veränderungen in den Talauen der jeweiligen Landschaftsräume. Aufgrund dieser naturbedingten Unterschiede setzten die anthropogenen Aktivitäten wie Rodung, Ackerbau oder Bergbau in den einzelnen Landschaftsräumen zu unterschiedlichen Zeiten und mit unterschiedlicher Intensität und Wirksamkeit ein. So tritt im Untersuchungsgebiet vor allem der Gegensatz zwischen dem siedlungsfeindlichen hohen Schwarzwald und den Gunstlagen des Oberrheintieflandes auf. Um 


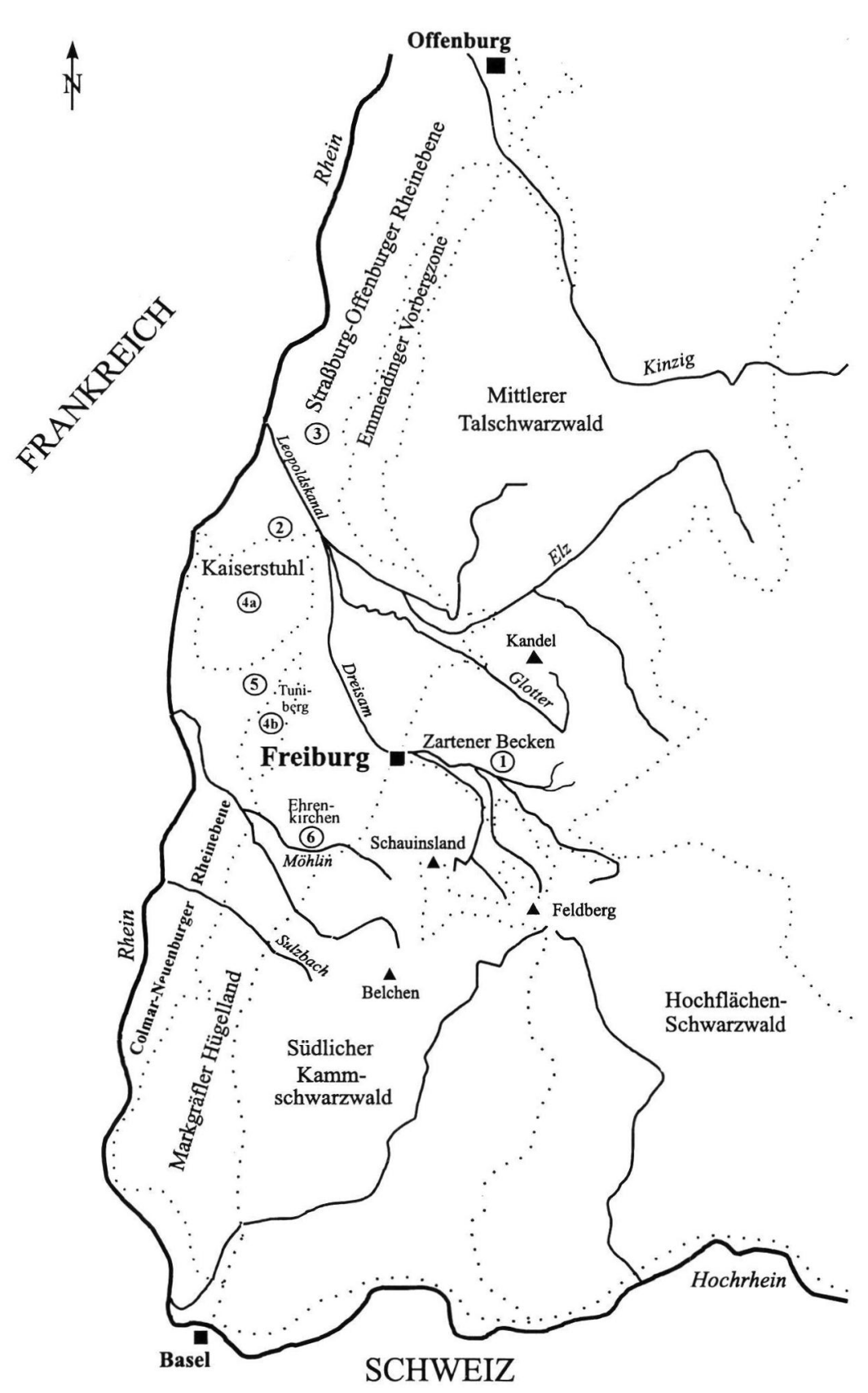

(1) Zartener Becken (49) Kaiserstuhl

$\begin{array}{ll}\text { (2) Endingen } & \text { (1D) Tuniberg }\end{array}$

(3) Rheinhausen

(5) Wasenweiler Ried

(6) Ehrenkirchen

$0 \quad 10 \mathrm{~km}$

Abb. 1: Übersichtskarte südliches Oberrheintiefland und Schwarzwald mit den Untersuchungsgebieten.

Fig. 1: Map showing the southern Upper Rhine Valley and the Black Forest with the investigated areas. 
diese anthropogenen Umwelteinflüsse wie auch die milieubedingten Umweltveränderungen des Holozäns erfassen zu können, wurden Aufschluß- und Bohrarbeiten an repräsentativen Stellen der verschiedenen naturräumlichen Einheiten durchgeführt. Dabei konzentrierten sich die Forschungsarbeiten auf das Einzugsgebiet der Elz von den Quellmulden bis zur Mündung in den Rhein. Verstärkte Untersuchungen fanden zudem im Einzugsgebiet der Dreisam, vor allem im Zartener Becken sowie im Gebiet Kaiserstuhl - Tuniberg statt (Abb. 1).

Ein zentrales Anliegen des Schwerpunktprogramms ist es, verfeinerte Methoden für die zeitliche Auflösung und Einordnung der natürlichen Archive zu erlangen. Dafür wurden bei den Forschungsarbeiten archäologische Funde, sedimentologisch-geomorphologische und pedologische Indikatoren ausgewertet sowie die Pollenanalyse eingesetzt. Hinzu kamen zahlreiche ${ }^{14} \mathrm{C}$-Datierungen von organischen Proben (zumeist Holzkohle, s. Danksagung, Kap. 6).

\section{Aktivitätsphasen im Zartener Becken zur Zeit der Kelten und Römer}

Günstige Aufschlüsse entstanden 1995 und 1996 durch Brücken- und Wegebau an der Landstraße zur B31-Ost im Zartener Becken (Abb. 1), z. B. von der L 126 südwestlich Kirchzarten über den Hagenbach und den Krummbach. Seit 1997 konnten zudem Aufschlüsse entlang eines Rohrleitungsgrabens parallel zur L 126 aufgenommen werden, der von der Niederterrasse zwischen dem Gewann Fischerrain durch die holozänen Auen des Krummbachs und des Hagenbachs führt. Die Auenbereiche zeichnen sich dort durch ein bewegtes Kleinrelief aus. Besonders fallen die Terrassenstufen der holozänen Talaue auf sowie kleine langgezogene Rücken bzw. mulden- und kerbförmige Vertiefungen auf den Auenniveaus. Insofern war der Schnitt durch die Krummbachbzw. Hagenbachaue wichtig für die Untersuchung des Aufbaus und der Genese des Auenreliefs.

\subsection{Aufbau und Altersstellung der Auenniveaus}

Für einen Brückenbau der L 126 über die B31-Ost wurde Ende 1996 ein tiefer Einschnitt (ca. 8 m) in die Niederterrasse im Gewann Rotmatte bei Zarten angelegt. Die Niederterrasse (370 $\mathrm{m}$ ü. NN) wird hier von einer ca. $25 \mathrm{~cm}$ mächtigen Auenlehmschicht überdeckt. Bei $20 \mathrm{~cm}$ u. GOF konnte ein fast vollständiger, unverrollter latènezeitlicher
Topf geborgen werden. Auf dem Gewann Rotacker wurden bereits zahlreiche archäologische Funde aus der Latènezeit gemacht. Hier wird auch im Vorfeld von Tarodunum (spätlatènezeitliches Oppidum) eine unbefestigte latènezeitliche Siedlung vermutet (mdl. Mitt. Dr. R. DeHn, Landesdenkmalamt Freiburg). Der Fund belegt eine Überflutung der Niederterrassenfläche noch nach dem ersten bzw. zweiten vorchristlichen Jahrhundert. Die Höhenlagen der Auensedimente geben zusätzlich einen Hinweis auf die außerordentliche Stärke des dazu notwendigen Hochwassers, denn die etwa $10 \mathrm{~m}$ entfernt gelegene Niederterrassenkante weist eine Höhe von über $3 \mathrm{~m}$ über dem rezenten Auenniveau auf! Es ist aber durchaus möglich, daß das Flußbett der Dreisam zur Zeit der Überschwemmung höher lag und die Eintiefung und damit der große Höhenunterschied zur Niederterrasse erst in der Neuzeit erfolgte. Vergleichbare Beobachtungen liegen aus den Schwarzwaldtälern südlich von Freiburg vor, z. B. aus dem Norsinger Grund (Zoluinger \& BUCHER 1989).

Das oberste Niveau der holozänen Krummbachaue (368,0 $\mathrm{m}$ ü. NN) liegt nahe der Niederterrasse und wird von einem $35 \mathrm{~cm}$ mächtigen lehmigen Auenboden aufgebaut, der wiederum von einem $75 \mathrm{~cm}$ mächtigen Schotterkörper in lehmiger Matrix unterlagert wird. Dabei handelt es sich um ein Auensediment, das aus umgelagerten Niederterrassenschottern bzw. holozänen Flußschottern mit z. T. großen Blöcken und Lehm besteht. Darunter folgen die ungestörten Niederterrassenschotter.

Das zweite Krummbachauenniveau $(366,5 \mathrm{~m})$ repräsentiert den Auentyp mit $120 \mathrm{~cm}$ Auenlehmdecke ohne Geröllanteil, in dem auch Ziegelreste gefunden wurden. Das unterste Krummbachauenniveau $(366,0 \mathrm{~m})$ hingegen zeigt den Wechsel zwischen Feinmaterial und Schottern entsprechend der Lage des Krummbachs (Abb. 2). Ein ehemaliges Bachbett des Krummbachs wurde angeschnitten (Querprofil in Abb. 2). Es kann auch oberflächlich noch verfolgt werden. Eine Holzkohlenprobe aus einer Sandlage unter dem Krummbachschotter und dem älteren Auenlehm ergab ein Alter von $617 \pm 54$ Jahren (Hd 19301). Es handelt sich also um eine hochmittelalterliche Ablagerung (cal AD 1305 bis 1410), die der Auenlehmphase 7 zuzuordnen ist (Tab. 1).

Der Aufschluß zwischen Krummbach und Hagenbach zeigt eine fast $90 \mathrm{~cm}$ mächtige Auenlehmdecke (Abb. 3), in der bei $26 \mathrm{~cm} \mathrm{u.} \mathrm{GOF} \mathrm{ei-}$ 


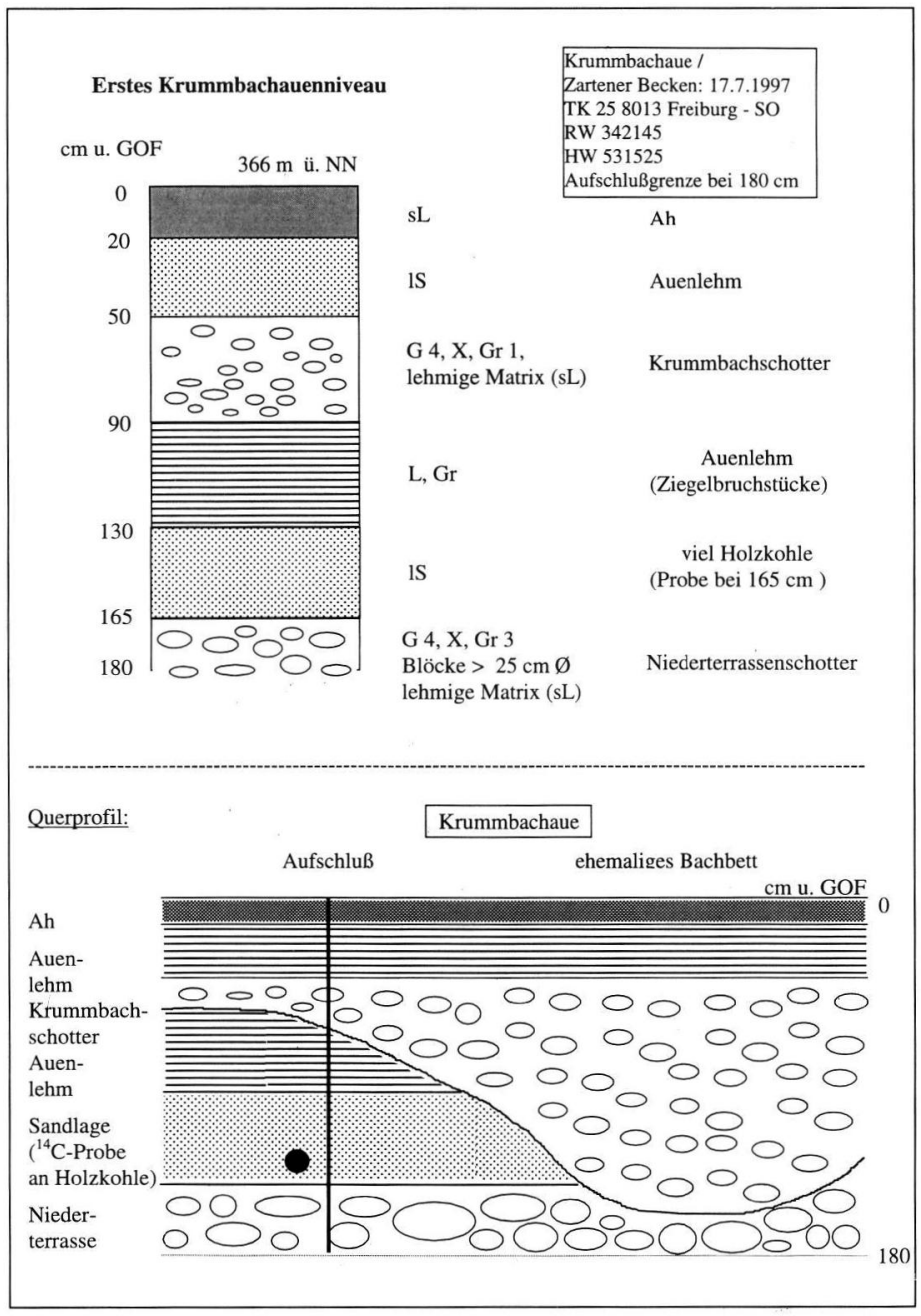

Abb. 2: Erstes Krummbachauenniveau, Zartener Becken, dargestellt an einem Aufschluß (oben) und im Querprofil (unten).

Fig. 2: First alluvial level of the Krummbach-river, Zartener Becken.

ne Scherbe, wahrscheinlich römischen Alters, gefunden wurde. Bei $42 \mathrm{~cm}$ u. GOF trat ein Holzkohleband auf. Die Probe daraus enthielt jedoch zu wenig ${ }^{14} \mathrm{C}$ für eine Datierung. Die obere Auenlehmdecke ist durch ein Sandband in $30 \mathrm{~cm}$ Tiefe in zwei Pakete gegliedert. Die untere Auenlehmdecke bis $88 \mathrm{~cm} \mathrm{u.} \mathrm{GOF} \mathrm{hat} \mathrm{einen} \mathrm{geringen} \mathrm{Anteil}$ an Geröllen und ist sehr arm an Holzkohle. Darauf folgt bis $130 \mathrm{~cm}$ u. GOF ein Schotterpaket in lehmiger Matrix. Die Gerölle (bis zu $15 \mathrm{~cm}$ im Durchmesser) stammen aus dem Niederterrassenschotter und wurden im Holozän umgelagert. Darunter kommen bis zur Aufschlußgrenze bei $270 \mathrm{~cm} \mathrm{u}$. GOF ungestörte Niederterrassenschotter mit Blöcken (über $30 \mathrm{~cm}$ im Durchmesser) vor. Im Aufschluß Hagenbach-Brücke (Abb.4) reicht die Auenlehmdecke aus schluffigem Lehm, in der mittelalterliche und neuzeitliche Scherben (Keramik aus dem 17. Jh. n. Chr.) vorkamen, bis $40 \mathrm{~cm} \mathrm{u}$. GOF. An manchen Stellen traten auch Sand- und Schotterlinsen im Auenlehm auf. Im Liegenden folgt zwischen 40 und $75 \mathrm{~cm} \mathrm{u}$. GOF ein dunkelbrauner schluffig-toniger Lehm mit eingelagerten Schottern und sogar Blöcken. Der hohe Humusanteil läßt auf einen begrabenen Ah-Horizont schließen. Das besondere an dieser Sedimentschicht war der Gehalt an Scherben, die zu einem römischen Topf zusammengesetzt werden konnten. Farblich abgesetzt von dieser dunkelbraunen Schicht folgt ein gelbliches, mit Geröllen und Blöcken angereichertes Auensediment aus tonigem Lehm, in dem in unterschiedlicher Tiefe Holzkohlestückchen vorkamen. Die Datierung von Holzkohle in einer Tiefe zwischen 110 und $130 \mathrm{~cm} \mathrm{u}$. GOF ergab ein Alter von $3189 \pm 60 \mathrm{BP}(\mathrm{Hd}$ 18301), eine weitere Probe $92 \mathrm{~cm}$ u. GOF wies hingegen ein weit höheres Alter von $7191 \pm 70$ BP (Hd 18298) auf. Der Altersunterschied erklärt sich aus einer Umlagerung bzw. rinnenhaften Eintiefung des älteren Schotterkörpers; die tiefer u. GOF liegende Holzkohle ist einer jüngeren Schotterfüllung in einer Rinne oder einem Kolk zuzuordnen. Die Basis des Aufschlusses besteht schließlich aus Schottern und Sanden des Würmglazials.

Südlich des Hagenbachs setzt sich eine ähnliche Abfolge der Auensedimente bezüglich der Korngrößenzusammensetzung fort. Als repräsentatives Beispiel wird der Aufschluß Hagenbachaue am Rande der Niederterrasse herangezogen. Unter einem 40 - $60 \mathrm{~cm}$ mächtigen humosen Auenlehm (Ah-Horizont) folgt ein toniger Lehm mit Steinen, die etwa $40 \%$ der Aufschlußfläche ausmachen. Darunter folgen bis in $160 \mathrm{~cm}$ Tiefe umgelagerte Schotter mit Ziegelresten, die wegen ihrer groben Herstellung vermutlich aus eisenzeitlicher Produktion stammen. An der Basis sind bis $440 \mathrm{~cm}$ Tiefe die Niederterrassenschotter aufgeschlossen. 
Tab. 1: Holozäne Auenlehmphasen im Mittleren und Südlichen Schwarzwald und Oberrheintiefland.

Tab. 1: Holocene phases of alluvial sedimentation in the Central and Southern Black Forest and Upper Rhine Valley.

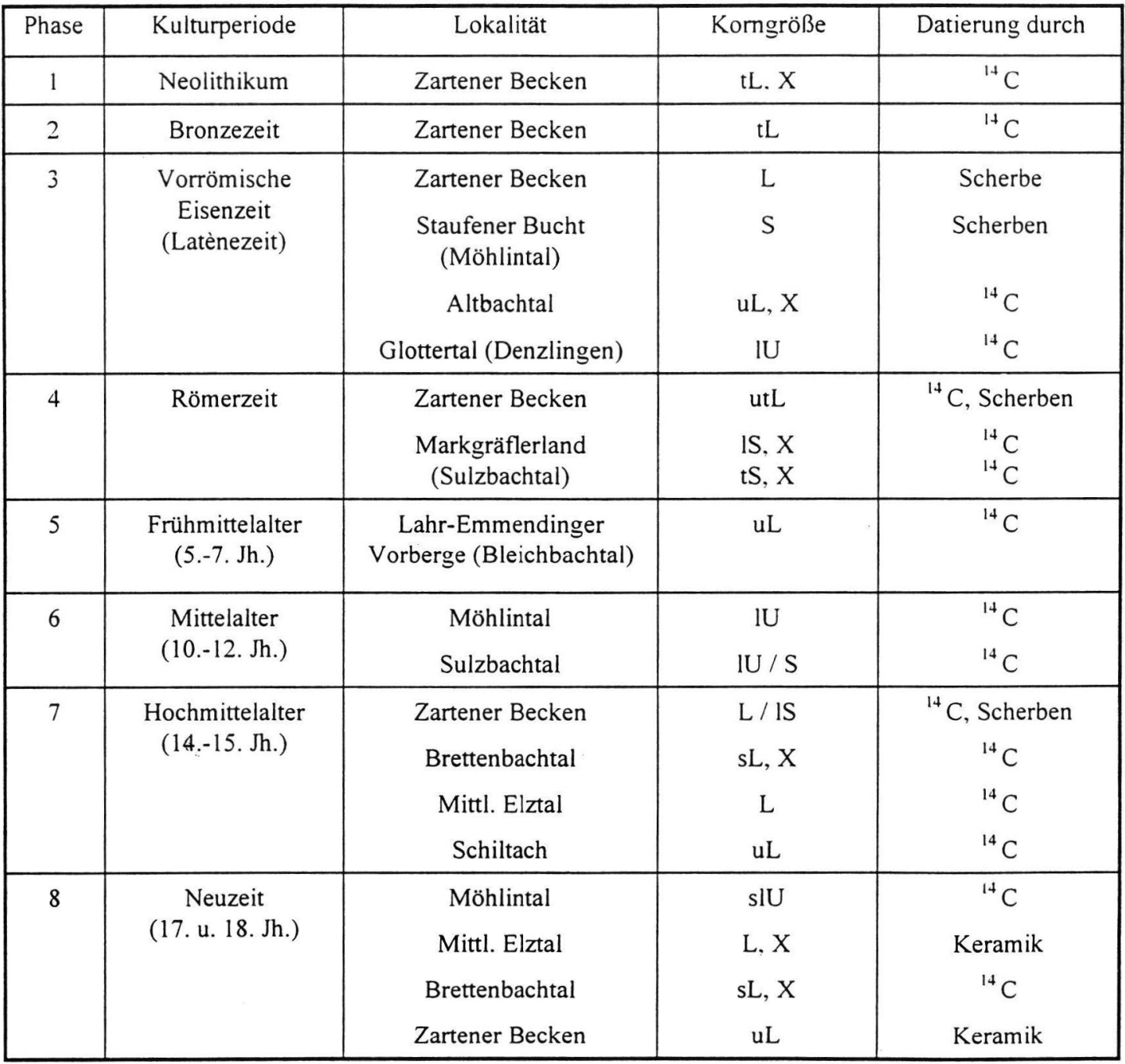

\subsection{Phasen und Ursachen der verstärkten} Auensedimentation

Die Auensedimente in den Aufschlüssen des Zartener Beckens sind durch 14C-Datierungen und archäologische Funde verschiedenen Auenlehmphasen zuzuordnen (Tab. 1). Das älteste Auensediment des Holozäns, ein toniger Lehm reich an Blöcken und Steinen, stammt danach aus dem Atlantikum (Zeitscheibe II). Eine verstärkte Auensedimentation ist jedoch erst für die Zeitscheibe III auszumachen. Sie beginnt in der Bronzezeit (Auenlehmphase 2) und tritt vor allem in der vorrömischen Eisenzeit (Auenlehmphase 3) und in der Römerzeit auf (Auenlehmphase 4, Tab. 1). Als Ursache für die hohe Sedimentfracht mit
Grobmaterial wird die starke Entwaldung der Hänge angenommen. Diese muß bereits zu vorrömischer Zeit eingesetzt haben, da der Bauund Feuerholzverbrauch in der latènezeitlichen Siedlung Tarodunum ungeheure Mengen an Holz verschlungen hat. Von den entwaldeten und teilweise ihrer Bodendecke entblößten Hängen konnten erhebliche Mengen an Lockermaterial abgetragen werden. Ähnliche Ablagerungen sind auch von der Westabdachung des Schwarzwaldes bekannt, z. B. im Altbachtal nordöstlich Freiburg. Dort fand im ausgehenden Subboreal eine Schotterakkumulation statt, worauf Lößablagerungen folgten, die nach ${ }^{14} \mathrm{C}$-Datierungen an Holzkohle in der Latènezeit zwischen $2150 \pm 60$ 


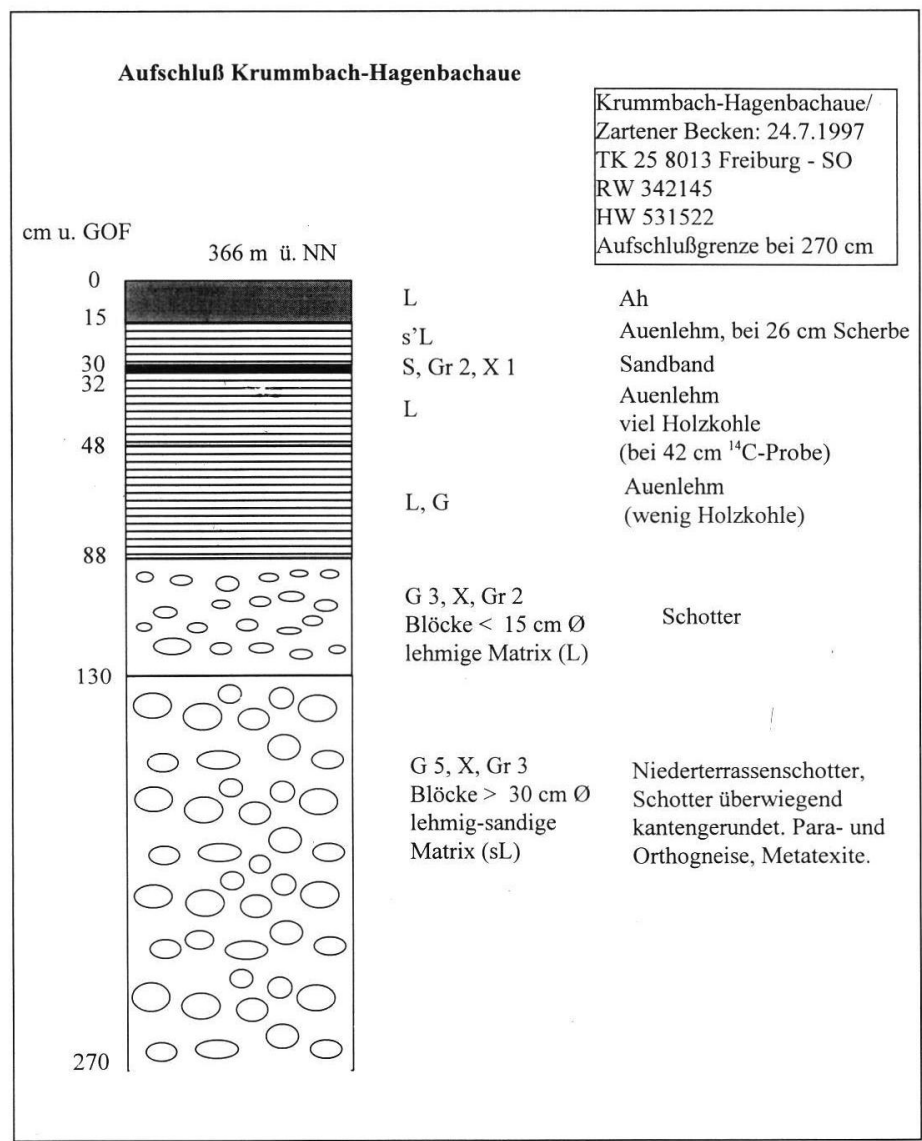

Abb. 3: Aufschluß Krummbach-Hagenbachaue, Zartener Becken.

Fig. 3: Alluvial sequence in the Krummbach-Hagenbach-alluvial plain, Zartener Becken.

$\mathrm{BP}$ und $2290 \pm 70 \mathrm{BP}$ im Altbachtal abgelagert wurden (Mä́cKel \& ZOLLINGER 1995, S. 95).

Die Scherben des römischen Topfes im Aufschluß Hagenbachbrücke (Abb. 4) weisen auf eine römerzeitliche Siedlung im Zartener Becken hin (villa rustica?). Da die Scherben unverrollt waren, können sie nicht weit transportiert worden sein. Als Ursache für die Ablagerung ist ein Großereignis anzunehmen, bei dem schotterreiches Hochflutsediment abgelagert wurde. Gegen Ende der Römerzeit ist eine Aufgabe der Siedlungen und eine Abwanderung der Bevölkerung anzunehmen. Allmählich erholte sich der Wald wieder und bildete einen wirksamen Schutz gegenüber Abtragung. Es herrschte somit eine Phase geomorphologischer Stabilität mit Bodenbildung an den Hängen und in den Talauen. Während der alamannischen Landnahmezeit sind im Zartener Becken - im Gegensatz zum Oberrheintiefland und der Vorbergzone - keine Siedlungsaktivitäten anzutreffen. Erst im Hochmittelalter erfolgte eine dichtere Besiedlung und landwirtschaftliche Nutzung, die sich auch in dem häufigen Auftreten von Auensedimenten (Auenlehmphase 7) widerspiegelt.

Auffallend sind an den Aufschlüssen Hagenbach-Brücke und entlang der Rohrleitung in der Hagenbachaue die schotterreichen Auensedimente (Abb. 3 und 4). Sie weisen auf häufige katastrophale Hochwasser hin, bei denen die Talauen stark überprägt wurden. Ähnliche Hochwasserereignisse katastrophalen Ausmaßes ereigneten sich im Juli 1987 und im Dezember 1991. Sie lieferten einen Einblick in die aktuelle fluviale Geomorphodynamik der Talauen (MÄCKEL \& RÖHRIG 1991, MÄCKEL \& ZoluINGER 1995): Die Ergebnisse des strömenden Hochflutwassers waren Erosionsformen wie Rinnen und Rillen, Abrißkanten und Auskolkungen. Als Akkumulationserscheinungen traten geschlossene Sand- und Steindecken mit Blöcken auf oder eine lockere Schotterstreu. Bei normalem Frühjahrshochwasser werden diese Grobmaterialablagerungen wieder von Feinmaterial überdeckt. Ein dichter Wechsel von katastrophalen und normalen Hochwassern führt zur Bildung eines schotterreichen Auensediments, das vor allem für Täler der hohen Mittelgebirge mit großer Reliefenergie typisch ist. Gefördert wird die hohe Groblastführung der Flüsse durch die oben bereits erwähnte intensive Nutzung der Hänge. Eine weitere Durchmischung von Steinen und Feinmaterial geschieht durch Pflugarbeit im Oberboden der Talauen.

\section{Landschaftswandel in der Vorbergzone}

Die Untersuchungen zum Landschaftswandel in der Vorbergzone des nördlichen Markgräfler Hügellandes und der südlichen Freiburger Bucht konzentrierten sich auf die Täler des Sulzbachs und der Möhlin (Abb. 1). Besonders wichtig für die paläoökologische Einordnung der Ablagerungsprozesse waren die reichhaltigen Holzkohlelagen und die archäologischen Funde in 
Aufschluß Hagenbach-Brücke, Zartener Becken

cm u. GOF

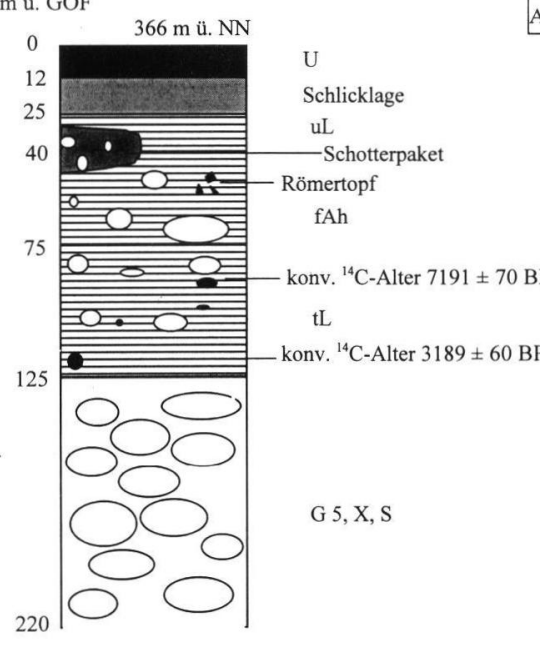

Hagenbachbrücke/

Zartener Becken: 26.1.1996

TK 258013 Freiburg - SO

RW 342145

HW 531520

Aufschlußgrenze bei $225 \mathrm{~cm}$

Ah

Auenlehm

(Neuzeitliche Keramik

17. Jh. n. Chr.)

Bodenbildung in schluffigtonigem Lehm mit Schottern, wenig gr. Blöcke

Block-/steiniger Auenlehm, (Holzkohle)

Niederterrassenschotter u. Sande

Abb. 4: Aufschluß Hagenbach-Brücke, Zartener Becken.

Fig. 4: Alluvial sequence Hagenbach-bridge, Zartener Becken.

den Baugruben im Möhlintal bei Ehrenkirchen und Bollschweil.

\subsection{Aufbau und Alter der Sedimente im Möhlintal}

Im Industriegebiet Niedermatten ergaben sich folgende Sedimentlagen (Abb. 5): Die Auenlehmdecken aus überwiegend schluffigem Lehm oder lehmigem Schluff sind an den meisten Stellen zwischen 50 und $100 \mathrm{~cm}$ mächtig. Darunter erscheinen Sand-Schlick-Wechsellagen und Schotterbänder. Der geschlossene Schotterkörper beginnt zwischen 100 und $150 \mathrm{~cm} \mathrm{u}$. GOF und ist nach oben hin von Schlickbändern durchzogen. Die Aufschlußgrenzen liegen bei $230 \mathrm{~cm}$ u. GOF. Zur Möhlin hin (Richtung SSW) wurde der Schotterkörper durch eine Rinne oder einen Mäanderbogen ausgeräumt. Die Eintiefung wurde später mit Sand- und Schlicklagen wieder aufgefüllt. In diesen Sand-Schlick-Wechsellagen treten in etwa 75 bis $145 \mathrm{~cm} \mathrm{u.} \mathrm{GOF} \mathrm{Holzkohlebänder} \mathrm{auf}$ (Abb. 5). Einzelne Holzkohlestücke hatten eine Größe von bis zu $4 \mathrm{~cm}$ im Durchmesser. Aus jedem Holzkohleband wurden Proben für die ${ }^{14} \mathrm{C}$ Datierung entnommen. Die Proben aus dem obersten Holzkohleband, das bei $55 \mathrm{~cm} \mathrm{u.} \mathrm{GOF}$ den Auenlehm (lehmiger Schluff) von den SandSchlick-Wechsellagen trennt, ergab ein Alter von
$1052 \pm 44 \mathrm{~J}$. BP bzw. cal. AD 975 bis 1025 (Hd-18275). Die Proben der unteren Holzkohlebänder in dem Sand-Schlick-Körper hatten ein Alter zwischen $946 \pm 31$ und $979 \pm 29 \mathrm{~J}$. BP bzw. cal. AD 1025 bis 1165 (Hd-18370 bis 18372). Eine weitere Probe (165 cm u. GOF) im Schotterkörper der aufgefüllten Rinne ergab ein Alter von $1045 \pm 39$ J. BP bzw. cal. AD 9851025. Nach den Datierungen gehört der Auenlehm zur mittelalterlichen Sedimentationsphase (Auenlehmphase 6, Tab. 1), die auch an anderen Stellen der Vorbergzone durch Keramik und ${ }^{14} \mathrm{C}$ Daten nachgewiesen wurde (MÄCKEl 1997). Neuzeitliche Auenlehme (Auenlehmphase 8) wurden ebenfalls in der Neumagen-Möhliner Niederung, und zwar im Neubaugebiet Ehrenstetten, gefunden (Mä́cKel \& RÖHRIG 1991, S. 306). Die ${ }^{14}$ C-Datierung einer Holzkohleprobe an der Basis des Auenlehms (slU) $80 \mathrm{~cm} \mathrm{u}$. GOF ergab ein ${ }^{14} \mathrm{C}$-Alter (BP) von $240 \pm 38$ Jahren (K-3095). In der Baugrube bei Bollschweil konnte der neuzeitliche Auenlehm vom mittelalterlichen Auenlehm im Liegenden durch Scherbenfunde unterschieden werden.

Im Aufschluß Niedermatten wurden in der oberen Lage der Schotter eine Schlacke und weitere Holzkohlestücke gefunden (Abb. 5). Die Schlacke wurde von Prof. Dr. J. Отто, Institut für Mineralogie, Petrologie und Geochemie der Universität Freiburg, durch eine Dünnschliffanalyse und Untersuchung im Auflichtmikroskop als Fayalith bestimmt und stellt eine Verhüttungsschlacke dar. Der Schotterkörper kann mit den sandigen Schottern korreliert werden, die in einem naheliegenden Kanalisationsgraben aufgeschlossen waren (Abb. 6). Dort lagen in $160 \mathrm{~cm}$ Tiefe Scherben, darunter ein großes Stück. Nach der Bestimmung von Dr. R. DeHn und Prof. Dr. G. FingerLin (Landesdenkmalamt Baden-Württemberg, Archäologische Denkmalpflege) gehören die Scherben zu einem Gefäß aus der Spätlatènezeit (2. bis 1. Jh. v. Chr.). Aufgrund der horizontalen Ablagerung und des Erhaltungszustandes der großen Scherben im Schotterkörper ist anzunehmen, daß diese nicht oder nur kurz im fluvialen Milieu verlagert wurde. Demnach wurde der Schotterkörper wohl im jüngeren Subatlantikum 
abgelagert. Diese Annahme wird durch die ${ }^{14} \mathrm{C}-$ Datierung von Holzkohle gestützt, die aus einer Sandschicht zwischen zwei Schotterlagen, $115 \mathrm{~cm}$ u. GOF, entnommen wurde. Das ${ }^{14}$ C-Alter (BP) betrug $2260 \pm 55$ Jahre, das Kalenderalter lag bei 400 - 190 v. Chr. (KI 3640).

\subsection{Einwirkung des Menschen auf die Landschaftsgenese innerhalb der Zeitscheibe III}

In den Tälern der Möhlin und des Sulzbaches wurde im ausgehenden Subboreal bzw. im frühen Subatlantikum eine verstärkte Schotterakkumulation beobachtet (MÄCKEL \& ZOLLINGER 1995). Wahrscheinlich geht der geomorphologische Umbruch zu vermehrter Akkumulation auf die Eingriffe des Menschen in der vorrömischen Eisenzeit (ab 800 v. Chr.) und besonders in der Latènezeit ( $a b 500 \mathrm{v}$. Chr.) zurück. Wirtschaftliche Aktivitäten aus dieser Zeit wie die Anlage von Siedlungen und der Bergbau bedingen einen großen Holzbedarf. Dazu kommt die landwirtschaftliche Nutzung des Umlandes (Rodung, Waldweide). Die weit verbreitete Abholzung der Hänge hatte eine verstärkte Abspülung von Lockermaterial zur Folge, das in den Hangfußzonen oder in den Talauen abgelagert wurde. Als Ursache für den einschneidenden Landschaftswandel werden die Bevölkerungszunahme und ein feuchteres und kühleres Klima (Abnahme der mittleren Jahrestemperatur um 2 bis $3^{\circ} \mathrm{C}$ nach Rudloff 1980) angenommen. Die Auswertung von Fundkarten (u. a. von SchmidT \& STÜLPNAGEL 1965, MäHling 1982) zeigt eine Zunahme der Siedlungen sowie eine Ausdehnung und Intensivierung der Anbauflächen, u. a. durch besseres Gerät, um mehr Nahrungsmittel produzieren zu können.

Eine weitere Ursache der starken Schotterakkumulation ist in den Bergbauaktivitäten der Latènezeit zu suchen. Diese These wird unterstützt von anthropogenen Schwermetallanreicherungen in den entsprechenden Auensedimenten (FOELLMER, Hoppe \& Dehn 1997). Einen zusätzlichen Beleg für den latènezeitlichen Bergbau liefert die Schlacke im Schotterkörper in Verbindung mit dem latènezeitlichen Scherbenfund (Abb. 5 und 6).

Eine spürbare Auswirkung auf die Hangabtragung und Flußdynamik hatten auch die Eingriffe der Römer in den Landschaftshaushalt, wie die Auswertung von Aufschlüssen im Sulzbachtal bei Sulzburg ergaben. Hier wurden die 2 bis $2,5 \mathrm{~m}$ mächtigen holozänen Schotterpakete aus vorrömischer Zeit, die sich durch eine Bodenbildung in sand- und kiesreichen Auensedimenten abgrenzen ließ, während der Römerzeit von einer neuen Schotterdecke überschüttet (ZOLLINGER \& MÄCKEL 1989, MÄCKEL \& ZOLLINGER 1995). Die zeitliche Einordnung erfolgte durch ${ }^{14} \mathrm{C}$-Analysen von Holzkohleproben, die aus Sandschichten zwischen den Schottern entnommen wurden und ein Kalenderalter von $20 \mathrm{v}$. Chr. - $250 \mathrm{n}$. Chr. bzw. $40 \mathrm{v}$. Chr. - 355 n. Chr. (KI 2413 und 2412) ergaben. Die römerzeitliche Sedimentschüttung wird wiederum durch eine Ruhephase mit Bodenbildung von den mittelalterlichen Auensedimenten getrennt, die nach ${ }^{14} \mathrm{C}$-Datierungen von $\mathrm{Holz}$ und $\mathrm{Holz}$ kohle ein Kalenderalter zwischen 1055 und 1295 n. Chr. (KI-2415 u. 2416) ergaben.

Die verstärkte Akkumulation in den Talauen steht im Zusammenhang mit der zunehmenden Waldzerstörung, die auf den erhöhten Bedarf an Brenn- und Bauholz für den Erzbergbau, die Verhüttung bzw. den Siedlungsbau zurückzuführen ist. Auf den tiefgreifenden Landschaftswandel an der Westabdachung des Südschwarzwalds seit der Römerzeit weisen verschiedene montanarchäologische Arbeiten hin (u. a. ZimmermanN 1990, Steuer 1990, Steuer \& Zimmermann 1993a,b; LuTfFMANN 1996).

Als Beispiel für eine aktive Hangdynamik dient der Aufschluß im Neubaugebiet Britzingen (Markgräfler Vorbergzone zwischen Badenweiler und Sulzburg). Dort finden sich in der Bergfußzone nordöstlich des Ehebachs 2 bis $3 \mathrm{~m}$ mächtige Lößlehmablagerungen, die nach einer ${ }^{14} \mathrm{C}$-Datierung von eingelagerten Holzkohlestücken um $3130 \pm 140 \mathrm{BP}$, also in der Bronze- bis Hallstattzeit (jüngeres Subboreal) abgelagert wurden (MäCKEL \& RÖHRIG 1991). Ebenfalls aus der Zeit vom Jüngeren Subboreal bis zum Älteren Subatlantikum stammen die von Zollinger (1990) aufgenommenen Kolluvien (sandhaltiger Schwemmlöß) am Ausgang der Norsinger Schlucht bei Ehrenstetten (Tab. 2).

\section{Die Lößsohlentäler und Schwemmfächer am Kaiserstuhl und Tuniberg}

Die Untersuchungen beschäftigen sich mit dem Aufbau der Lößsohlentäler und Schwemmfächer sowie den Erosions- und Akkumulationsprozessen an den Hängen und im Talbereich des Kaiserstuhls und Tunibergs (Abb. 1). Im Mittelpunkt steht die Zeitscheibe III (1500 v. Chr. bis $500 \mathrm{n}$. Chr.), kulturgeschichtlich der Zeitraum von der Bronzezeit bis zur Römerzeit (Kap. 1). Das charakteristische Sediment in den Hangkolluvien dieser Gebiete ist der Schwemmlöß. Er ist aqua- 


\section{Profil Gewann Niedermatten, Ehrenstetten \\ Lage: TK 25:8012, RW 3404775, HW 5310025}

$\mathrm{cm}$

u. GOF N $234 \mathrm{~m}$ ü. NN

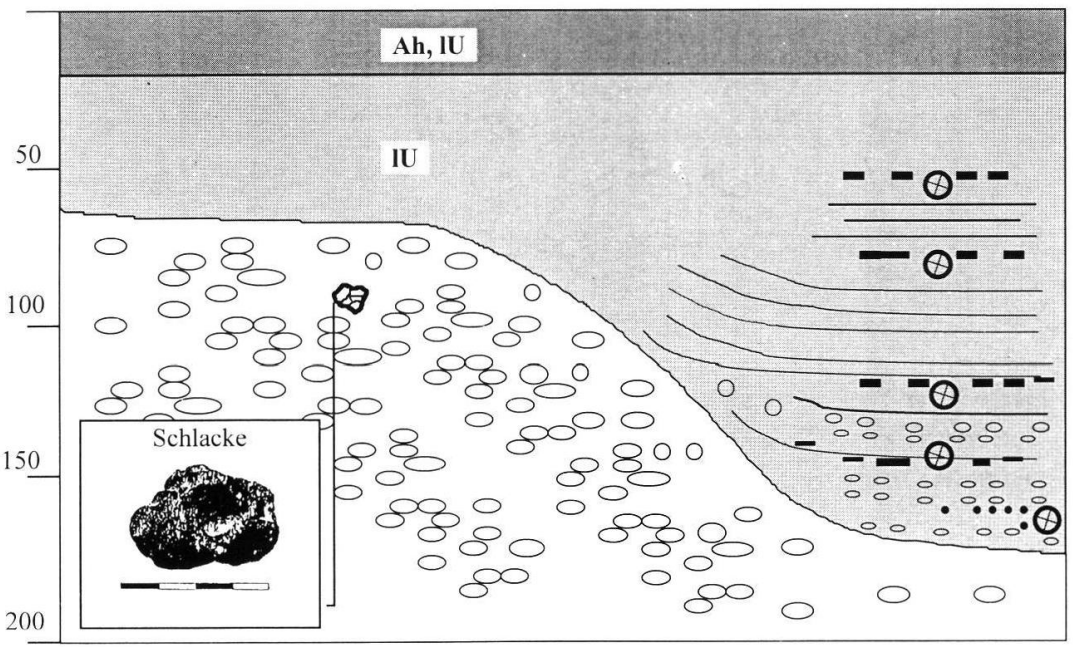

${ }^{14} \mathrm{C}(1)$

Wechsellage Sand / Schlick

${ }^{14} \mathrm{C}(3)$

${ }^{14} \mathrm{C}(4)$

${ }^{14} \mathrm{C}(5)$

Abb. 5: Aufschluß 1 (Baugrube) im Gewann Niedermatten, Ehrenkirchen.

Fig. 5: Alluvial section 1 in Niedermatten, Ehrenkirchen.

tisch umgelagert und im Unterschied zum Primärlöß kalkarm oder entkalkt, geschichtet und instabil. Die Korngrößenzusammensetzung bleibt jedoch weitgehend erhalten, und der Schluffanteil dominiert jeweils. Im Schwemmlöß bzw. Kolluvium kommen jedoch auch gröbere Materialien wie Sand, Grus oder Schnecken vor. Schwemmlöß bzw. Kolluvium kann auch heute noch bei Starkregenfällen entstehen. Vor allem an den Böschungen der Rebterrassen und an den Hohlwegen wurden verschiedene Abtragungsprozesse und Erosionsformen im Löß aufgenommen (MÄCKEL 1997).

Bei den Tälern des Kaiserstuhls und Tunibergs handelt es sich nicht um Kastentäler oder Lößschluchten, sondern um eine eigene Form (Friedmann \& Mäckel 1998). Die Täler sind überwiegend Sohlentäler, die durch anthropogene Überprägung (teils Terrassierung) mit einem scharfen Knick in die Hangbereiche übergehen. Der Begriff Lößsoblental bzw. seitlich terrassiertes Lößsohlental beschreibt die Talform besser.

\subsection{Bohrprofile im Spührenlochtal bei Oberbergen}

Bis heute ist nicht geklärt, unter welchen Umständen und innerhalb welcher Zeiträume die Ausbzw. Umformung der Täler im Kaiserstuhl zu Löß- sohlentälern vor sich ging. Im Gewann Spührenloch bei Oberbergen im Kaiserstuhl wurden daher mehrere Bohrungen niedergebracht, um den Aufbau und die Genese der Lößsohlentäler zu erforschen.

Die Profilabfolgen der fünf Bohrungen im unteren Talabschnitt waren recht unterschiedlich je nach Lage im Querprofil. An den Talseiten wurde schon nach etwa $3 \mathrm{~m}$ u. GOF der Primärlöß erreicht. Bei zwei Bohrungen am Rande des Rückhaltebeckens konnte das Tal vollständig durchteuft werden, wobei zwischen $720 \mathrm{~cm}$ bis 745 u. GOF im Grundwasserniveau die Verwitterungszone des Anstehenden bzw. periglazialer Schutt erreicht wurde (Abb. 7). Die Vergleyung durch periodische Staunässe beginnt bei den tieferen Bohrungen 3, 4 und 5 bei etwa $300 \mathrm{~cm}$ u. GOF. Bohrung 4 ( $273 \mathrm{~m}$ ü. NN), die in der Talmitte in einer Rinne liegt, soll hier detailliert erläutert werden (Abb. 8): Als Folge der Rebumlegungen und des 1977 gebauten Rückhaltebeckens besteht die heutige Oberfläche bis 130 $\mathrm{cm}$ u. GOF aus anthropogen aufgebrachtem Material. Darunter tritt der humose A-Horizont des früheren Oberbodens (fAh) auf. Dann folgt bis $67 \mathrm{~cm} \mathrm{u}$. GOF Kolluvium mit einzelnen steinigen und sandigen Lagen, nur unterbrochen von einem $4 \mathrm{~cm}$ mächtigen humosen Band bei 251 bis 


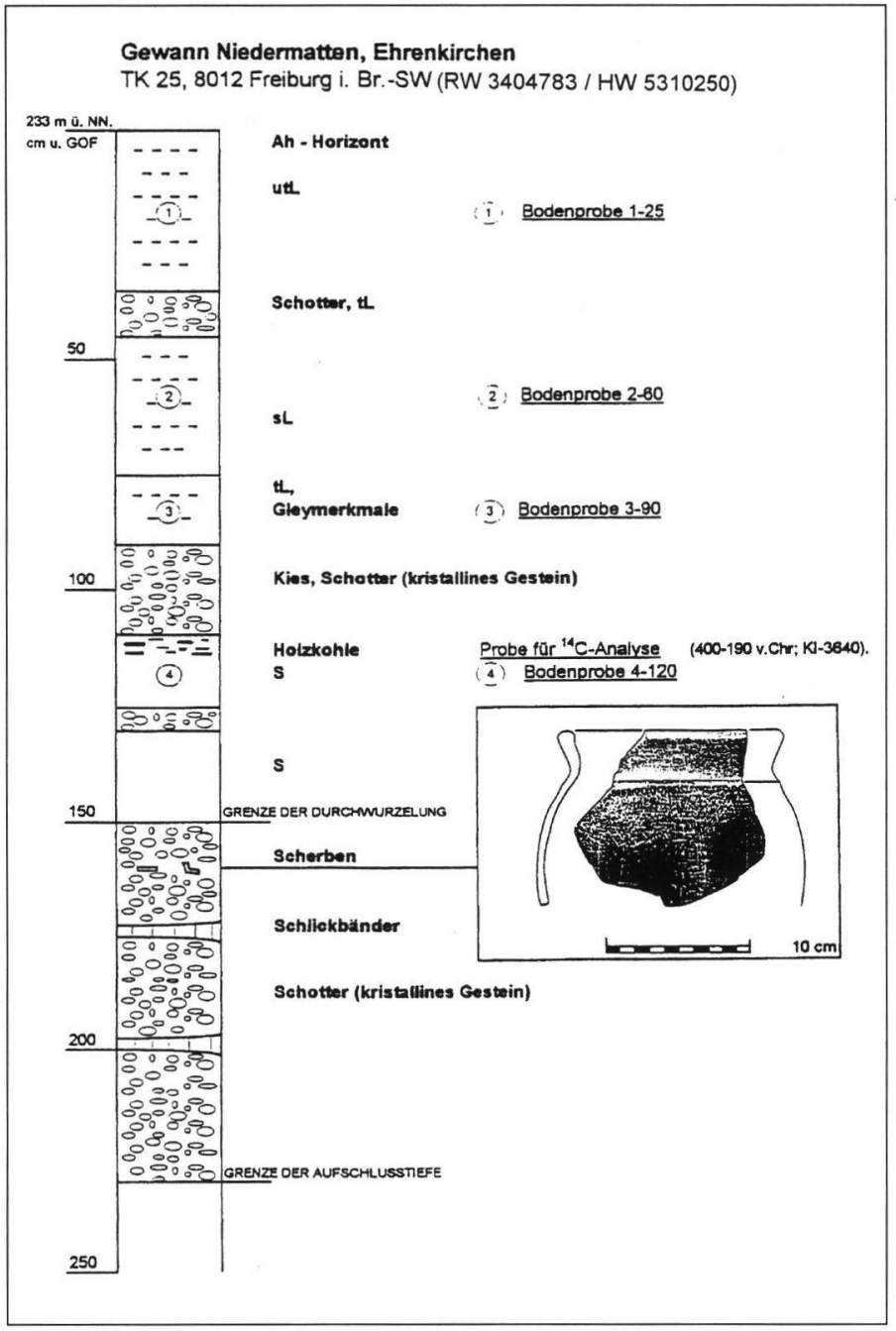

Abb. 6: Aufschluß 2 (Kanalisationsgraben) im Gewann Niedermatten, Ehrenkirchen.

Fig. 6: Alluvial section 2 in Niedermatten, Ehrenkirchen.

$255 \mathrm{~cm}$ u. GOF. Dieses Bändchen enthielt zahlreiche organische Reste, von denen Proben genommen wurden. Von 467 bis $470 \mathrm{~cm} \mathrm{u}$. GOF tritt ein weiteres Torfband auf, worauf bis $564 \mathrm{~cm} \mathrm{u}$. GOF wieder Kolluvium folgt. Bei $530 \mathrm{~cm}$ u. GOF lagen ein Knochenstück und gebrannter Lehm. In der darunterliegenden Lößlehmschicht, die nach unten durch eine $11 \mathrm{~cm}$ mächtige Sand- und Steinlage begrenzt wird, wurde Ziegelbruch gefunden. Danach folgt wieder Kolluvium bis 565 $\mathrm{cm}$ u. GOF. In $628 \mathrm{~cm}$ u. GOF wurde im Kolluvium ein bronzezeitliches Scherbenbruchstück entdeckt. Bei 656 bis $665 \mathrm{~cm} \mathrm{u}$. GOF tritt eine weitere torfige Schicht auf, aus der Proben für die ${ }^{14} \mathrm{C}$ Datierung entnommen wurden. Das Alter dieser
Torfprobe liegt bei $2465 \pm 26 \mathrm{BP}$ (Abb.8). Bis $690 \mathrm{~cm} \mathrm{u.} \mathrm{GOF} \mathrm{ist} \mathrm{Kol-}$ luvium und Lößlehm anzutreffen und von 690 bis $717 \mathrm{~cm} \mathrm{u}$. GOF eine weitere Torflage direkt über einer mächtigen Steinschicht mit sandiger Matrix. Aus der Torflage wurde wiederum eine Probe für die ${ }^{14} \mathrm{C}$-Datierung entnommen, die ein Alter von $2530 \pm 27 \mathrm{BP}$ ergab (Abb. 8). Der Steinhorizont besteht aus kantigen Steinen in einer breiigen Sandmatrix, die sich im Grundwasserniveau befindet und sich bis zur Endtiefe in $855 \mathrm{~cm} \mathrm{u}$. GOF fortsetzt. Alle Steine stammen von Essexiten, einem Subvulkanit, der im ganzen Tal das Muttergestein bildet. Daraus kann geschlossen werden, daß es sich hier wohl um eine periglaziale Deckschicht oder eine basisnahe Schuttdecke handelt.

Der schematisierte Aufbau des Lößsohlentales Spührenloch (Abb. 7) zeigt, daß der Primärlöß, der den anstehenden Essexit überlagert, in der Talmitte ausgeräumt wurde. Diese Rinne oder Vertiefung ist in ihren unteren Bereichen teilweise durch Schutt- und Steinlagen, überwiegend jedoch durch Kolluvium, verfüllt. Die sich anschließenden Talhänge sind terrassierte Rebflächen bis zu einer Höhe von ca. 300-385 m, ab welcher sich Laubwald anschließt. Das Talquerprofil ist asymmetrisch mit einem steileren Osthang und einem flacheren Westhang. Das Lößsohlental wurde an mehreren Stellen auf ganzer Länge durchbohrt und durch Pürckhauerbohrungen an den Seiten ergänzt. Über archäologische Funde konnte eine frühe keltische Siedlungsaktivität in diesem Gebiet nachgewiesen werden (s. u.). Die verschiedenen Torfschichten zeigen Ruhephasen an, in denen eine Konsolidierung der Geomorphodynamik erfolgte. Die Ursache liegt wahrscheinlich in der abnehmenden wirtschaftlichen Nutzung dieses Raumes und damit in der geringeren anthropogenen Beeinflussung des Sedimentationsgeschehens. Die ${ }^{14} \mathrm{C}$-Datierungen ermöglichen eine absolute zeitliche Einordnung dieser Phase und können den Beginn der Umformung des Spührenloches zum Lößsohlental festlegen. Der 
Tab. 2: Holozäne Phasen der Kolluvienbildung im Oberrheintiefland und Schwarzwald.

Tab. 2: Holocene phases of colluvial sedimentation in the Upper Rhine Valley and in the Black Forest.

\begin{tabular}{|c|c|c|c|c|}
\hline Phase & Kulturperiode & Lokalität & Korngröße & Datierung durch \\
\hline 1 & Neolithikum & Kaiserstuhl (Endingen) & U & Scherben \\
\hline 2 & Bronzezeit & $\begin{array}{l}\text { Markgräflerland (Ehebachtal) } \\
\text { Kaiserstuhl } \\
\text { (Spührenloch) (Ellenbuch) }\end{array}$ & $\begin{array}{c}\text { IU (Lößlehm) } \\
\text { IU (Lößlehm) IU } \\
\text { (Lößlehm) }\end{array}$ & $\begin{array}{l}\text { Scherbe } \\
{ }^{1+} \mathrm{C}\end{array}$ \\
\hline 3 & $\begin{array}{l}\text { Vorrömische } \\
\text { Eisenzeit } \\
\text { (Latènezeit) }\end{array}$ & $\begin{array}{l}\text { Kaiserstuhl (Spührenloch) } \\
\text { Westabdachung (Altbachtal) } \\
\text { (Norsinger Grund) }\end{array}$ & $\begin{array}{l}\text { IU (Lößlehm) } \\
\text { uL, X } \\
\text { IU, S }\end{array}$ & $\begin{array}{c}{ }^{14} \mathrm{C}, \text { Scherbe } \\
{ }^{14} \mathrm{C} \\
{ }^{14} \mathrm{C}\end{array}$ \\
\hline 4 & Römerzeit & Kaiserstuhl (Spührenloch) & IU & Scherben \\
\hline 5 & $\begin{array}{l}\text { Alamannische } \\
\text { Landnahme- und } \\
\text { Ausbauzeit } \\
\text { (5.-8. Jh.) }\end{array}$ & $\begin{array}{c}\text { Lahr-Emmendinger. } \\
\text { Vorberge (Bleichbachtal) }\end{array}$ & $\mathrm{uL}$ & ${ }^{14} \mathrm{C}$ \\
\hline 6 & $\begin{array}{l}\text { Ottoner- und } \\
\text { Merowingerzeit } \\
(11 .-13 . \text { Jh. })\end{array}$ & Kaiserstuhl (Ihringen) & IU (Lößlehm) & ${ }^{14} \mathrm{C}$ \\
\hline 7 & $\begin{array}{l}\text { Hochmittelalter } \\
\text { (14./15. Jh.) }\end{array}$ & Mittl. Eltztal & L & strat. Einordn. \\
\hline 8 & $\begin{array}{c}\text { Neuzeit } \\
\text { (17. u. 18. Jh.) }\end{array}$ & $\begin{array}{c}\text { Mittl. Elztal } \\
\text { Markgräflerland (Dellen im } \\
\text { Löß) }\end{array}$ & $\begin{array}{l}\mathrm{L} \\
\mathrm{IU}\end{array}$ & $\begin{array}{l}{ }^{14} \mathrm{C} \\
\text { rezenter } \mathrm{Bt}\end{array}$ \\
\hline
\end{tabular}

unterste Torfhorizont ist direkt auf die Schuttsohle der Talrinne aufgewachsen und zeigt durch das ${ }^{14}$ C-Alter von $2530 \pm 27 \mathrm{BP}$ an, daß die Ausräumung des Tales vor diesem Zeitpunkt abgeschlossen sein mußte. Ab diesem Zeitpunkt beginnt die Umformung des Kerbtales in ein Lößsohlental durch verstärkte Hangabtragung und Materialakkumulation in der Talsohle. Der zweite Torfhorizont ergab ein ${ }^{14} \mathrm{C}$-Alter von $2465 \pm 26$ BP, womit die $30 \mathrm{~cm}$ Sediment zwischen erstem und zweitem Torfhorizont in ca. 65 Jahren umgelagert wurden, was eine jährliche Sedimentationsrate von $0,46 \mathrm{~cm}$ ergeben würde. Jedoch erfolgt die Abtragung und Sedimentation, wie auch heute noch zu beobachten, nicht gleichmäßig und kontinuierlich, sondern eher unregelmäßig bei besonderen oder einmaligen Ereignissen, wie z. B. nach Starkregen. Die Torfhorizonte datieren somit beide in die vorrömische Eisenzeit bzw. in die Hallstattkultur. Die Kelten, die zu dieser Zeit im Kaiserstuhl siedelten, haben dabei wahrscheinlich zum ersten Mal Teile des Waldes im Tal und an den Hängen gerodet und als Weide oder Ackerfläche genutzt.

Bei Bohrung 3 (274,5 m NN), $22 \mathrm{~m}$ westlich von Bohrung 4 (siehe Abb. 7), wurde $370 \mathrm{~cm} \mathrm{u.} \mathrm{GOF}$ eine hallstattliche Scherbe gefunden, die auch auf frühe Siedlungsaktivitäten der Kelten im Kaiserstuhl während der Hallstattzeit (ca. 750 - 450 v. Chr.) hindeuten. Bei den Rebumlegungen vor 20 Jahren wurde im Spührenloch 33 Fundstücke aus der Spätlatènezeit (ca. 450 - 10 v. Chr.) entdeckt, die auf die Existenz eines Gehöfts im Tal schließen lassen (WeBer-JENISCH 1995). Römische Fundstücke, die von DEHN (1984) in einem Aufschluß am Rückhaltebecken gefunden wurden, belegen zudem römische Siedlungsaktivitäten in diesem Gebiet. 


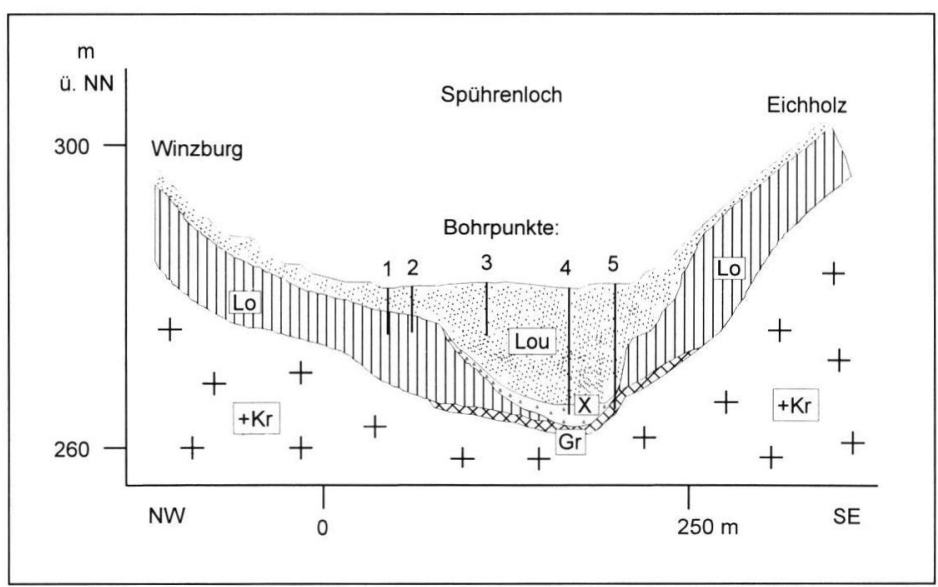

Abb. 7: Schematisierter Aufbau des Lößsohlentales Spührenloch bei Oberbergen, Kaiserstuhl.

Fig. 7: Schematic cross-section through the loess-valley Spührenloch near Oberbergen, Kaiserstuhl.

\subsection{Der Sedimentaufbau der Sohlentäler bei Bickensohl und im Tuniberg}

Im Ellenbuchtal westlich Bickensohl konnte ein $5 \mathrm{~m}$ tiefes Profil erbohrt werden. Bis $107 \mathrm{~cm} \mathrm{u}$. GOF fand sich anthropogen aufgebrachtes Material. Darunter begann schon der Grundwasserschwankungsbereich mit regelmäßiger Bleichung und Rostfleckung. Zwischen 136 und $154 \mathrm{~cm} \mathrm{u}$. GOF lag ein fossiler Ah-Horizont mit leicht erhöhtem Humusgehalt, so auch zwischen 214 und $234 \mathrm{~cm} \mathrm{u}$. GOF. Leider kam bis dahin kein ${ }^{14} \mathrm{C}-\mathrm{da}-$ tierbares Material vor. Erst im darunter liegenden Kolluvium wurden bei $266 \mathrm{~cm}$ u. GOF eine Holzkohlenlage und Knochenreste gefunden. Darunter nahm die Feuchte stark zu, und das Grundwasserniveau war nahe, bevor die Bohrung bei $500 \mathrm{~cm} \mathrm{u}$. GOF aufgrund technischer Probleme abgebrochen werden mußte. Beim aufgetretenen Kolluvium handelt es sich durchgehend um Lößlehm, an drei Stellen von sandig-grusigen Lagen unterbrochen. Die ${ }^{14} \mathrm{C}$-Datierung der Holzkohle in $266 \mathrm{~cm}$ Tiefe erbrachte ein Alter von $2642 \pm 50$ Jahre BP (Hd-19302) bzw. cal. BC 820-795 (895770). Demnach wurde das Kolluvium an der Wende der Bronzezeit zur vorrömischen Eisenzeit abgelagert. Eine erhöhte Geomorphodynamik zu dieser Zeit mit verstärkter Abspülung des Lösses und Ablagerung in den Talsohlen konnte bereits an anderen Stellen des Untersuchungsgebietes festgestellt werden (s. u.).

Weitere Bohrungen wurden in einem Lößsohlental des Tunibergs südwestlich von Waltershofen durchgeführt. Die Bohrung in der Nähe des Hofs Wippertskirch erreichte $538 \mathrm{~cm}$ Tiefe. Zwischen
67 und $92 \mathrm{~cm} \mathrm{u.} \mathrm{GOF} \mathrm{fand} \mathrm{sich} \mathrm{ein}$ fossiler Ah, darüber bis zur Oberfläche junges anthropogenes Material. Unter dem fossilen Ah-Horizont folgte bis zur Aufschlußgrenze Kolluvium im Grundwasserschwankungsbereich, das bis zu einer Tiefe von $181 \mathrm{~cm} \mathrm{u}$. GOF mehrmals kleine Ziegelreste aufwies. Holzkohle kam regelmäßig in kleinen Mengen vor, die jedoch nicht datierbar war.

\subsection{Die Schwemmfächer am Rande des Kaiserstuhls}

Der Kaiserstuhl und der Tuniberg werden von einem fast geschlossenen Schwemmlößsaum unterschiedlicher Breite umgeben (SCHREINER 1958). Er ist besonders ausgedehnt durch die Schwemmfächer am Ausgang der Lößsohlentäler. So verbreitert sich der Schwemmlößsaum auf der Nordseite des Kaiserstuhls bei Königschaffhausen auf 800 m, auf der Südseite bei Ihringen sogar auf $1700 \mathrm{~m}$ (SCHREINER 1959: 88 u. Geol. Karte). Im neuen Industriegebiet von Endingen ist der Schwemmlöß, etwa 1,5 bis $2,5 \mathrm{~m}$ mächtig, in den Baugruben über dem Primärlöß zu finden. Bei Ihringen erreichen die Schwemmlößauflagen eine Mächtigkeit bis zu 5 m (SCHREINER 1996: 299). Neolithische und bronzezeitliche Funde in und unter den Schwemmlößdecken belegen die verstärkte Bildung des Schwemmlösses seit dem Neolithikum (Zeitscheibe II). Von solchen Funden unter 2 bis $4 \mathrm{~m}$ mächtigen Lößlehmdecken im Innern des Kaiserstuhls berichtet bereits LaIS (1934, 1935). SLEUMER (1934: 26) stellt mit Hilfe pollenanalytischer Untersuchungen den Lößlehm bei Wasenweiler in das Atlantikum. Insgesamt kann auf der Grundlage der bisherigen Funde davon ausgegangen werden, daß neben den Lößtälern im Kaiserstuhl auch die Schwemmfächer am Rande des Kaiserstuhls vollständige Archive bilden und somit Auskunft über den Landschaftswandel unter den Einwirkungen des Menschen geben. Als Beispiele werden Aufschlüsse in Baugruben in Ihringen (südlich des Kaiserstuhls) und in Endingen (nördlich des Kaiserstuhls) herangezogen.

In der Baugrube im Neubaugebiet von Ihringen wurden $160 \mathrm{~cm} \mathrm{u}$. GOF bzw. $180 \mathrm{~cm}$ u. GOF zwei durch Lößlehm getrennte Torfschichten gefunden. Nach der ${ }^{14} \mathrm{C}$-Datierung stammen beide aus dem frühen Mittelalter. Die untere weist ein Alter 


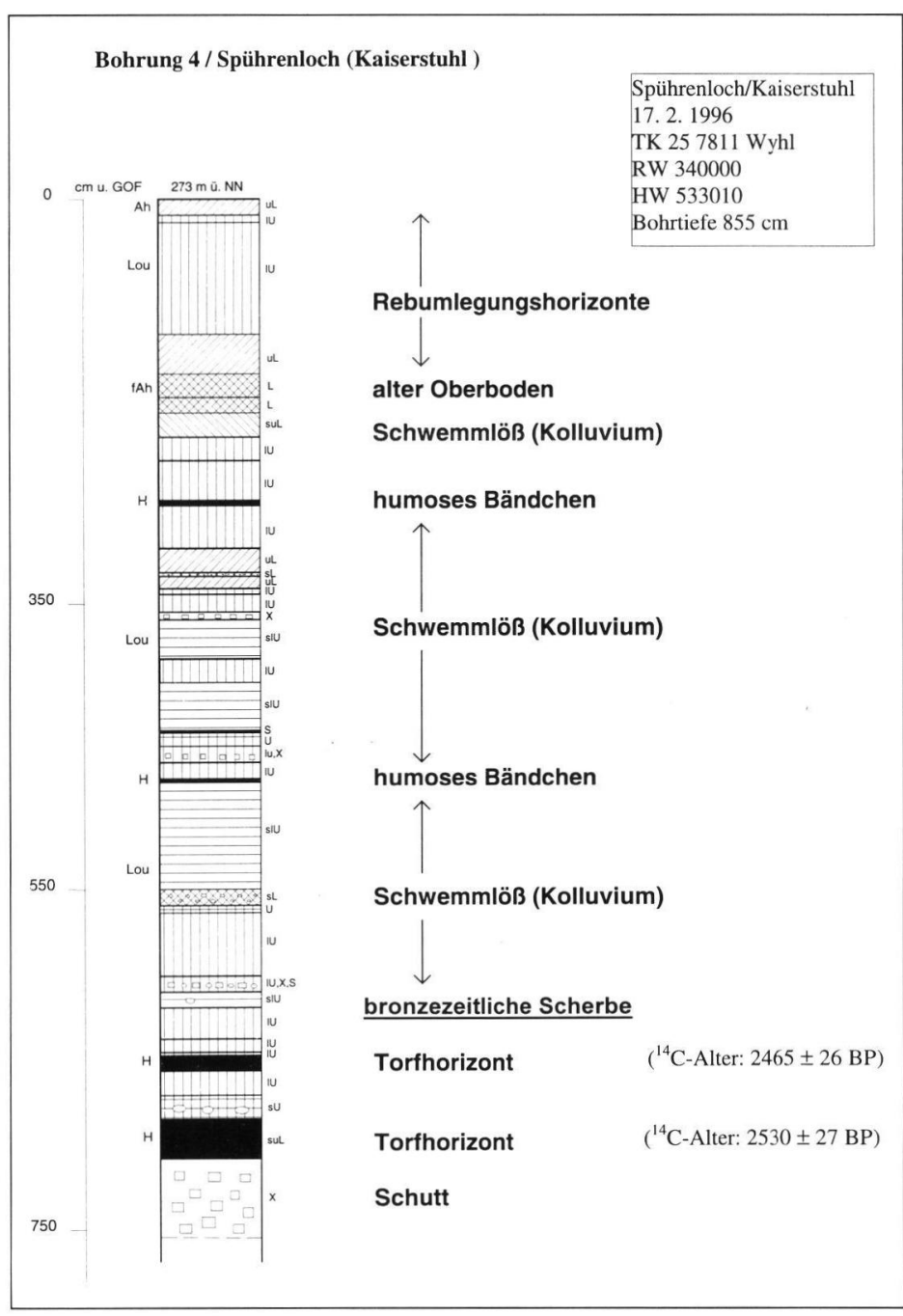

Abb. 8: Bohrung 4, Spührenloch, bei Oberbergen, Kaiserstuhl.

Fig.8: Stratigraphy of sediment core 4, Spührenloch near Oberbergen, Kaiserstuhl.

von $1297 \pm 28 \mathrm{BP}$ (Merowingerzeit), die obere eines von $1035 \pm 34 \mathrm{BP}$ (Ottonenzeit) auf (Tab. 2). Die Schwemmlößschicht dazwischen belegt die aktive Geomorphodynamik am südlichen Kaiserstuhlhangfuß zur Karolingerzeit.

Nordwestlich der Altstadt von Endingen wurde ein Aufschluß in einer Baugrube aufgenommen, in dem unter einer $160 \mathrm{~cm}$ mächtigen differenzierten Lößlehmdecke ein fossiler dunkelbrauner Bodenhorizont aus schluffigem Ton auftritt (Abb. 9). Dieser Boden war nur noch als Rest in zwei 1 $\mathrm{m}$ bzw. 2,5 m breiten Gruben erhalten, die mit unregelmäßiger Abgrenzung in den Primärlöß eingelassen sind. In diesen Vertiefungen fanden sich Keramik und Holzkohlestückchen. Die Keramik stammt von der Wauwiler Gruppe nach Dieckmann (1990, 1991), die zu Beginn des Jungneolithikums ( 4200 bis 4300 v. Chr.) im Kaiserstuhl lebte. Die Funde belegen eine Abtragung des damaligen schwarzerdeähnlichen Oberbodens bis auf die Reste in den beiden Gruben und eine nachfolgende verstärkte Akkumulation des Lößlehms.

\subsection{Auswertung der Befunde in den Lößlandschaften von Kaiserstuhl und Tuniberg}

Während sich die neolithischen Siedlungen im Kaiserstuhl, wie zu dieser Zeit allgemein üblich, eher auf Höhenrücken oberhalb der Täler befanden, erfolgte die Besiedlung der tieferen Talbereiche wohl erst durch die Kelten und die Römer. Im klimabegünstigten Lößsohlental Spührenloch im zentralen Kaiserstuhl kann jedoch von einer Siedlungskontinuität seit dem Neolithikum ausgegangen werden (Denn 1984). Die Bohrungen im Spührenloch zeigen damit von der Zersetzungszone des Anstehenden bis zur heutigen Oberfläche ein vollständiges Archiv, das insbesondere die paläoökologischen Bedingungen während der Zeitscheibe III (1500 v. Chr. - 500 n. Chr.) widerspiegelt. Neben natürlichen Faktoren wie Tektonik und Klima hat der Mensch in diesen Zeiten maßgeblich auf die Umwelt der Lößlandschaft eingewirkt. Die anfänglich im Neolithikum geringe Bevölkerungsdichte hat spätestens mit den Kelten in der Hallstatt-/Latènezeit bis zu den Römern stetig zugenommen. Den zunehmenden Eingriff in den Landschaftshaushalt unterstreichen die mächtigen Kolluvien im Inneren und am Rande des Kaiserstuhls sowie in den Lößlandschaften der Vorbergzone (Kap. 3).

Die Ursachen für die verstärkte Erosion von den Hängen und Akkumulation in den Tälern liegen wie in der lößbedeckten Vorbergzone im Zusammentreffen klimatischer Schwankungen mit anthropogenen Einwirkungen zu Beginn der Eisenzeit (800 v. Chr.). Mit der Bevölkerungszunahme 


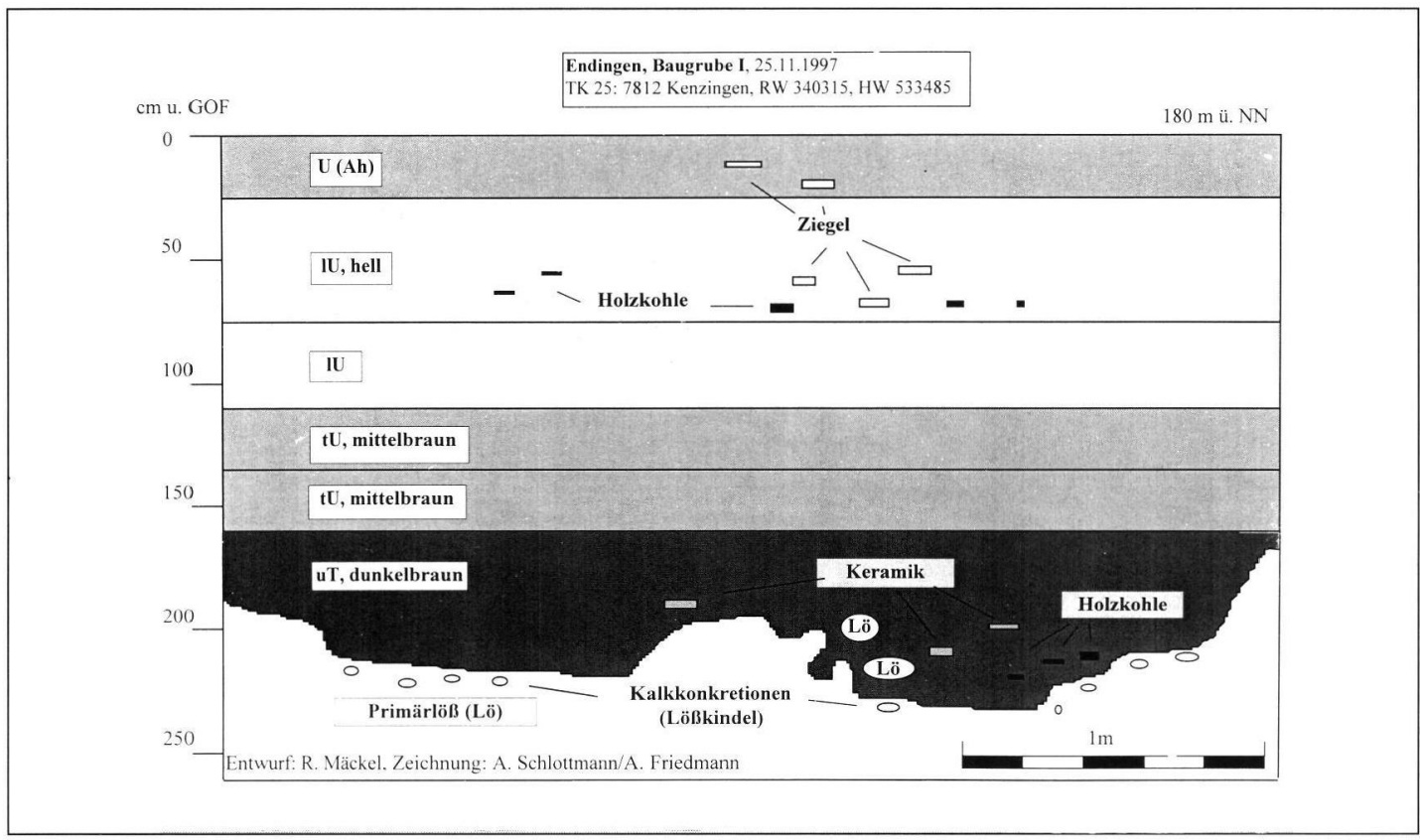

Abb. 9: Kolluvium über neolithischer Grubenfüllung in Endingen, nördliches Kaiserstuhlvorland.

Fig. 9: Colluvial cover over neolithic fill in Endingen, northern Kaiserstuhl foreland.

kam es zu einer Siedlungsverdichtung an Gunststandorten im Kaiserstuhl sowie zu einer Ausweitung der landwirtschaftlichen Nutzfläche an anderen Orten, um mehr Nahrung produzieren zu können. Hinzu kamen die besseren Geräte zu Beginn der Eisenzeit, mit denen die Anbauflächen gerodet und intensiver bearbeitet werden konnten. Ebenfalls mußten mehr Wälder für Brennund Baumaterial abgeholzt werden, was wiederum ungeschüitzte Flächen und nachfolgende Bodenerosion durch Wasser, aber auch durch Wind, verursachte. Die Abschwemmung des Lockermaterials von den Hängen in die (Kerb-)Täler führte zu einer großflächigen Auffüllung der Talsohlen mit Kolluvium. Dadurch entwickelten sich aus den ehemaligen Kerbtälern die heutigen Lößsohlentäler. Diese typische Talform des Kaiserstuhls und anderer Lößgebiete (Tuniberg, Vorbergzone) scheint somit primär das Ergebnis einer anthropogenen Einflußnahme auf das Erosions- und Akkumulationsgeschehen zu sein. Besonders deutlich wird dies nach DeHN (1984) in der Römerzeit (Terrassierung, Weinbau). Aufgrund der kräftigen Erosionsvorgänge sind ein Großteil der prähistorischen Besiedlungsspuren auf den Bergrücken und an den Hängen abgetragen. Die meisten archäologischen Funde sind in den Talsohlen oder Senken unter mehrere Meter mächtigen Kolluviumschichten $\mathrm{zu}$ finden. Die oberflächennahen
Schichten sind jedoch ohne archäologische Funde und spiegeln somit eine Siedlungsleere wider, die nur durch das Verständnis der anthropogenen Interaktion mit den geomorphologischen Prozessen erklärbar wird.

Eine ähnliche Genese des Lößsohlentals wie im Spührenloch finden wir z. B. in den Tälern von Bickensohl und Ellenbuch (südlicher Zentralkaiserstuhl) sowie Bucklinsbühl (Tuniberg). Die Täler des Kaiserstuhls erweisen sich damit als ideales Untersuchungsgebiet zur Auffindung vollständiger Archive. Die bisherigen Ergebnisse scheinen zu bestätigen, daß der Kaiserstuhl und die Vorbergzone schon früh im Neolithikum besiedelt wurden und eine Siedlungskontinuität über die Eisenzeit bis in die Römerzeit aufweisen, was mit einer starken anthropogenen Beeinflussung des Sedimentationsgeschehens einhergeht.

\section{Das Wasenweiler Ried und die Ostrheinproblematik}

Das $4 \mathrm{~km}^{2}$ große Wasenweiler Ried erstreckt sich in SW-NE-Richtung zwischen dem südlichen Kaiserstuhl und dem nordwestlichen Tuniberg (Abb. 1). Es ist ein reliktisches Niedermoor, da es seit 1920 systematisch entwässert wird. Dadurch wurden die obersten Schichten bereits zersetzt, so daß das Niedermoor heute eine geringere Mäch- 


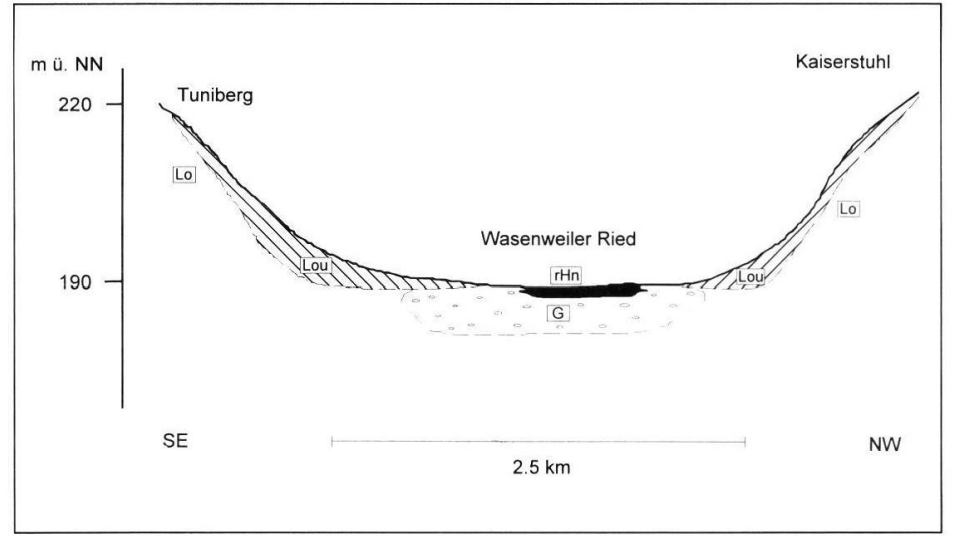

Abb. 10: Schematischer Querschnitt durch das Wasenweiler Ried.

Fig. 10: Schematic cross-section through the fen Wasenweiler Ried. haltung ist in der Nähe des Wasenweiler Riedes seit ca. 6500 BP nachweisbar. Eine Nutzung des Waldes durch Nieder- und Mittelwaldwirtschaft und eine daraus resultierende Beeinflussung der Baumartenzusammensetzung des Waldes durch den Menschen ist ab ca. 5500 BP zu erwarten. Ackerbau, sicher nachgewiesen durch Getreidepollen, setzte im direkten Umfeld des Wasenweiler Riedes ab 4500 BP ein, womöglich jedoch schon ab $5100 \mathrm{BP}$, was sekundäre Indikatorpollen (Ackerunkräuter) andeuten. Der Anteil der Kulturzeigerarten am Pollentigkeit besitzt als 1920. An seinen Rändern wird das Ried durch Schwemmlöß vom Kaiserstuhl und vom Tuniberg überlagert (Abb. 10). Die Entstehung des Riedes fällt ins Holozän und steht im Zusammenhang mit der Verlagerung des Ostrheins, der noch im Spätglazial den Kaiserstuhl auf der Ostseite umfloß (SCHreINER 1959, 1996).

Auch heute wird das Ostrheingebiet von einem auffallenden Rinnensystem markiert (KAYSER \& MÄ́kel 1994). An den Bohrprofilen war der Torf zwischen $1 \mathrm{~m}$ und 2,5 m mächtig. Darunter folgten nach einer Übergangsschicht von einigen Dezimetern die Ostrheinschotter, die hier bis $12 \mathrm{~m}$ mächtig sein können und randlich anstehen oder von Hanglöß überdeckt sind (Abb. 10). Im Wasenweiler Ried wurde zur Rekonstruktion der Vegetationsgeschichte von FRIEDMANN (1998) eine Pollenanalyse durchgeführt.

Das Pollendiagramm Wasenweiler Ried West umfaßt die gesamte Zeitscheibe II und den Beginn der Zeitscheibe III (ca. 7600 bis 3000 BP). In diese Zeit fällt der Beginn des Ackerbaus und die Seßhaftwerdung des prähistorischen Menschen in diesem Raum. Schließlich können im Abschnitt der Zeitscheibe III große Rodungen und eine Ausweitung des Offenlandes und Ackerbaus dokumentiert werden. Die wichtigsten Ereignisse für den Landschaftswandel sind im folgenden aufgeführt:

Es ist ein kontinuierlicher Rückgang des Waldes zugunsten des Offenlandes, nur einmal unterbrochen um ca. $3700 \mathrm{BP}$, festzustellen. Es traten somit zwei Phasen der Besiedlung auf, die von einer Wüstungsphase unterbrochen worden sind. Der Rückgang des Waldes ist überwiegend auf Rodungen durch den Menschen zurückzuführen. Waldweide und später auch allgemeine Weide- spektrum nimmt kontinuierlich bis an die Oberfläche zu, nur einmal unterbrochen während der Wüstungsphase um $3700 \mathrm{BP}$.

Die Untersuchungen im Wasenweiler Ried (West) haben somit zum ersten Mal für das südliche Oberrheingebiet wichtige pollenanalytische Ergebnisse zur Vegetations-, Ackerbau- und Siedlungsgeschichte erbracht.

Zusätzlich wurden im Randbereich des Wasenweiler Riedes mehrere Bohrungen durchgeführt, serscheide zwischen Rhein- und Dreisameinzugsgebiet zu klären. Der Dreisamschwemmfächer dehnte sich im Holozän aus (SCHreINER 1959; LEHMANN-CARPZOv et al. 1978) und dämmte das Ostende des Durchlasses zwischen Kaiserstuhl und Tuniberg $a b$, so daß die dortigen Gewässer nicht nach NE in die Freiburger Bucht abfließsen konnten.

Durch eine ${ }^{14} \mathrm{C}$-Probe am Übergang Torf Dreisamschwemmfächermaterial konnte das Vorrücken des Dreisamschwemmfächers bis an den Kaiserstuhl- und Tuniberg-Ostrand auf 4939 \pm 48 BP (cal BC 3775-3660) bestimmt werden (Friedmann 1998). Dies stellt die Ausdehnung des Dreisamschwemmfächers ins Subboreal (zur Zeit der Horgener Kultur), was eine Aktivitäts- und Erosionsphase im Oberlauf andeutet. Auch an anderen Stellen der Schwarzwaldabdachung (z. B. Möhlin, Sulzbach) erfolgte insbesondere im Subboreal eine Aufschotterung an den Unterläufen (Zollinger \& Mäckel 1989). Die verstärkte Schwemmfächerschüttung der Dreisam bewirkte einen Staueffekt im Wasenweiler Ried, wodurch der Torf ab dem Subboreal schneller wachsen konnte im Vergleich zum Atlantikum (Zeitscheibe II). um die fluviale Geomorphodynamik an der Was- 
Die Abschnürung und Verlandung des Ostrheins ist nicht gleichmäßig für alle Ostrheinrinnen verlaufen. So zeigen zwei datierte Basisproben aus verschiedenen Rinnen deutliche Altersdifferenzen (Friedmann 1998): eine Rinne aus dem Zentralbereich des Wasenweiler Riedes weist ein Basisalter von $7460 \pm 53$ BP (cal BC 6370-6185) auf, die zweite Rinne im nordöstlichen Bereich des Riedes am Auslaß zwischen Kaiserstuhl und Tuniberg hat ein ${ }^{14} \mathrm{C}$-Alter von $10727 \pm 98 \mathrm{BP}$ (cal BP 10815-10590). Die Hauptabschnürung des Ostrheins als großer dynamischer Rheinarm erfolgte somit schon im Spätglazial. Im Bereich des Wasenweiler Riedes südlich des Kaiserstuhls, wo die Rheinaue sehr nahe liegt, sind jedoch noch einzelne große Rinnen über mehrere tausend Jahre regelmäßig von Hochwassern des Rheines erreicht worden. Zeitlich zog sich somit die Entwicklung vom aktiven Ostrhein zu mehreren verlandenden Altarmen vom Alleröd bis ins Boreal, also über einen Zeitraum von über 3000 Jahren hin. Eine großflächige Vermoorung des Wasenweiler Riedes hat damit erst im Atlantikum begonnen, als der Torf über die Rinnen hinaus emporwuchs und flächenhaft weiterwachsen konnte. Es bildet sich ein Niedermoor (bzw. Verlandungsmoor) aus. Außerordentlich große Hochwasser des Rheins dürften jedoch gelegentlich bis in das späte Mittelalter einzelne Rinnen im westlichen Teil des Wasenweiler Riedes erfaßt haben (BANGERT 1958).

\section{Schlußfolgerungen}

Im Oberrheintiefland und an der Westabdachung des südlichen Schwarzwaldes wurden im Zuge des Straßen- und Siedlungsbaus sowie durch Bohrungen verschiedene holozäne Sedimentlagen ausgewertet. Sie bestehen zum Teil aus Grobmaterial (Schotterpaketen mit Blöcken) oder aus Feinsedimenten. Insgesamt lassen sich im Untersuchungsgebiet acht $14 \mathrm{C}$-datierte Auenlehmphasen unterscheiden (Tab. 1): 1. die neolithische Auenlehmphase (Zeitscheibe II), 2. die bronzezeitliche Auenlehmphase, 3. die latènezeitliche Phase, 4. die römerzeitliche Phase (1. bis 4. Jh.), 5. die Auenlehmphase der Landnahmezeit (5./6. Jh. n. Chr.) und des Siedlungsausbaus (7./8. Jh.), 6. die mittelalterliche Phase (10./12. Jh.), 7. die hochmittelalterliche (14./15. Jh.) und 8. die neuzeitliche Auenlehmphase (17./18. Jh.). Auch im Spätglazial gibt es Hochflutlehme (MäcKel 1998). Die Auenlehmphasen in diesem Aufsatz beziehen sich jedoch entsprechend dem Thema auf das Holozän und sind weitgehend auf den Einfluß des wirtschaftenden Menschen zurückzuführen. Zeitgleich $\mathrm{zu}$ den Auenlehmphasen kommen auch Phasen verstärkter Kolluvienbildung vor (Tab. 2).

Im Zartener Becken wurden entlang des Rohrleitungsgrabens parallel zur L 126 eine Reihe von Aufschlüssen aufgenommen, die einen flächenhaften Einblick in die Auenstratigraphie des Krummbaches und des Hagenbaches erlaubten. Dabei fiel auf, daß über den Niederterrassenschottern eine Schicht holozän umgelagerter Blöcke (z. T. kantengerundet) in lehmiger Matrix auftritt. Hier scheint es während eines holozänen Hochwasserereignisses zur Tiefenerosion (Rinnen- und Kolkbildung) und nachfolgender darin abgelagerter Materialakkumulation durch den Krumm- bzw. den Hagenbach gekommen zu sein. Es ist auch anzunehmen, daß bei diesem Großereignis Grobmaterial (Moränen- und Schuttmaterial) aus den Schwarzwaldtälern im Einzugsgebiet in das Zartener Becken transportiert wurden. Dieses herantransportierte Material wurde zusammen mit dem aus der Niederterrasse wiederaufgenommenen schließlich bei nachlassender Wasserfließgeschwindigkeit und Transportkraft des Flusses umgelagert. Darüber schließen sich Auenlehmdecken unterschiedlicher Mächtigkeit an, in deren oberes Drittel die rezente Bodenbildung reicht.

Das Auftreten von Ziegelbruchstücken (wahrscheinlich römischen oder eisenzeitlichen Alters) in den umgelagerten Niederterrassenschottern, der Fund eines römischen und eines latènezeitlichen Topfes und eines weiteren römischen Scherbenbruchstückes in den tieferen Auenlehmdecken bezeugen eine aktive Geomorphodynamik im Zartener Becken in der Zeitscheibe III (1500 v. Chr. bis 500 n. Chr.). Spätmittelalterliche und neuzeitliche Keramik aus den oberen Teilen der Auenlehmschichten und die Hochwasserereignisse der Jahre 1987 und 1991 belegen bis heute eine lebhafte fluviale Geomorphodynamik im Zartener Becken. Es fehlt jedoch eine aktive Phase zur Zeit der alamannischen Landnahme- und Ausbauzeit im Zartener Becken.

Trotz der naturräumlichen und milieuspezifischen Unterschiede der drei Untersuchungsgebiete (Zartener Becken, Vorbergzone und Kaiserstuhl/Tuniberg im Oberrheintiefland) lassen sich einige Gemeinsamkeiten bezüglich des anthropogenen Einflusses auf das Geoökosystem feststellen:

Nach einer Ruhephase im älteren Subatlantikum mit Bodenbildung ist während der Latènezeit (5. 
bis 1 . Jh. v. Chr.) verstärkte fluviale Aktivität festzustellen. Die Ursachen dafür liegen neben der Klimaänderung (kühler und feuchter) in den zunehmenden Eingriffen des Menschen in das Geoökosystem. Mit der Bevölkerungszunahme und Siedlungserweiterung erfolgte eine großflächige Entwaldung durch Rodung und Holzeinschlag (für Feuer- und Bauholz). Denn $(1983,1989)$ und Fingerlin (1991) weisen darauf hin, daß es sich bei den Siedlungen der Latènezeit um befestigte stadtähnliche Siedlungen handelt, für die ein sehr hoher Bedarf an Bauholz anzunehmen ist. Hinzu kommt die intensive Bearbeitung des Bodens mit verbesserten Geräten. Archäologische Funde und ${ }^{14} \mathrm{C}$-Daten belegen ebenfalls den Bergbau zu dieser Zeit mit bedeutsamem Einfluß auf Abtrag und Sedimentation. Aus dieser Zeit stammen auch die Auenlehmdecken im Zartener Becken und der Westabdachung des Schwarzwaldes, die der Auenlehmphase 3 zuzuordnen sind. Während der Römerzeit (1. bis 4. Jh. n. Chr.) sind die Eingriffe in den Landschaftshaushalt besonders einschneidend, vor allem durch den Siedlungs- und Straßenbau, die agrarische Landnutzung und den Bergbau. Mit dem Rückzug der römischen Bevölkerung im 3. bzw. 4. Jh. n. Chr. verringert sich die Bevölkerungsdichte erheblich, die landwirtschaftliche Nutzfläche wird kleiner und der Bergbau aufgegeben.

Weite Teile der entwaldeten Hänge am Schwarzwaldwestrand und in der Vorbergzone erhielten wohl wieder eine schützende Vegetationsdecke. In den Talauen deutet eine Bodenbildung auf die Konsolidierung der Flußdynamik hin. Insgesamt handelt es sich um Umweltveränderungen infolge eines nachlassenden menschlichen Einflusses, die ihren Niederschlag in den Sedimenten der Talaue gefunden haben. Daher führten die Aktivitäten während der vorrömischen Eisenzeit und der Römerzeit zu den schwerwiegendsten anthropogenen Veränderungen im Untersuchungsgebiet innerhalb der drei Zeitscheiben. Die lößbedeckten Vorberge des nördlichen Markgräfler Hügellandes und der Freiburger Bucht bilden ein günstiges Siedlungsgebiet. Funde von der prärömischen Eisenzeit und der Römerzeit im Sedimentationskörper der Täler belegen die intensive Nutzung dieses Raumes innerhalb der Zeitscheibe III. Dies führte zu einer verstärkten Erosion von den Hängen und zur Akkumulation in den Talauen, die nach ${ }^{14} \mathrm{C}$-Datierungen bereits in der Bronzezeit begonnen haben muß. Auffallend ist die Schotterakkumulation während der Latènezeit und während der Römerzeit im Möhlin- und Sulz- bachtal. Archäologische Funde sprechen dafür, daß die Ursache für die starke Schotterakkumulation in den Bergbauaktivitäten seit der Latènezeit zu suchen sind. Auenlehmablagerungen stammen aus allen Bildungsphasen. Besonders auffallend und reich an Holzkohlebändern sind die mittelalterlichen und neuzeitlichen Feinerdeablagerungen (Auenlehmphase 6 bis 8).

Im Kaiserstuhl und Tuniberg wurden mehrere Lößsohlentäler erbohrt, um der Frage nach der Entwicklung der Täler in klimabegünstigten Lößgebieten nachzugehen. Durch archäologische Funde, wie eine bronzezeitliche und eine hallstattzeitliche Scherbe in verschiedenen Schwemmlößhorizonten, konnte eine frühe Siedlungsaktivität im Kaiserstuhl nachgewiesen werden. Das Innere des Tunibergs ist im Vergleich zum Kaiserstuhl relativ fundarm. Die prähistorischen Siedlungen haben überwiegend am Hangfuß des Tunibergs gelegen, von denen aus auch die zentralen Teile des Tunibergs bewirtschaftet wurden. Die Täler des Kaiserstuhls waren ursprünglich Kerbtäler, oder sie wurden nachträglich ausgeräumt. Diese Ausräumung muß jedoch vor der Hallstattzeit (2530 BP) stattgefunden haben, was durch ${ }^{14} \mathrm{C}$-Datierungen an Torfhorizonten über dem Anstehenden im Spührenloch (Zentralkaiserstuhl) nachgewiesen werden kann. Danach wurden die Kerbtäler des Kaiserstuhls und Tunibergs durch abgespültes Kolluvium in ihrer Talsohle verfüllt, wodurch sie in Lößsohlentäler umgestaltet wurden. Am Kaiserstuhl hat eine Siedlungskontinuität vom Neolithikum bis zur Römerzeit geherrscht, wobei Rodungen, Siedlungs- und Straßenbau starke Eingriffe in den Landschaftshaushalt bedingten und zur Bildung eines mehrere Meter mächtigen Schwemmlößsaumes führten.

Die Entstehung des Wasenweiler Rieds steht im Zusammenhang mit der Ostrheinproblematik. Die Datierung von Torfproben beweist die frühere Annahme von SchreInER (1959, 1996), daß der Ostrhein als dynamischer Rheinarm bereits im Spätglazial aufhörte zu fließen. Jedoch zeigt das unterschiedliche Alter der großen Rinnen, daß eine Entwässerung des Ostrheins noch bis in das Boreal andauerte.

\section{Danksagung}

Die Untersuchungen zum holozänen Landschaftswandel wurden im Rahmen des DFGSchwerpunktprogramms durchgeführt, und zwar speziell im regionalen Teilprojekt „Die palökolo- 
gischen Umweltbedingungen im Oberrheintiefland und Schwarzwald im Neolithikum und zur Römerzeit - Fluviale Sedimente, Böden und Relief als Archive" (Ma 557/11). Die zum Vergleich herangezogenen Ergebnisse zur holozänen Flußgeschichte und Hangdynamik stehen im Zusammenhang mit dem DFG-Schwerpunktprogramm „Fluviale Geomorphodynamik im jüngeren Quartär", hier mit dem regionalen Teilprojekt ,Jungquartäre Fluß- und Hangdynamik im Schwarzwald und Oberrheintiefland“ (Ma 557/8). Die ${ }^{14} \mathrm{C}$-Datierungen wurden am Institut für Umweltphysik der Universität Heidelberg durchgeführt. Die Autoren danken vor allem Herrn Dr. Kromer für Analysen und die konstruktiven Ratschläge und Kommentare. Die ${ }^{14} \mathrm{C}$-Datierungen bis 1993 erfolgten im ${ }^{14} \mathrm{C}$-Labor der Universität Kiel. Die Sedimentanalysen (z. B. Korngrößenzusammensetzung) wurden im Institut für Physische Geographie der Universität Freiburg durchgeführt.

Besonderer Dank gilt den Mitarbeitern im DFGSchwerpunktprogramm, vor allem Frau A. Schlottmann, Herrn R. Schneider, Herrn M. Rauschkolb und Frau B. Weber für die Hilfe im Gelände und bei den Zeichenarbeiten. Frau S. Harrer tippte und korrigierte das Manuskript.

\section{Schriftenverzeichnis}

ANDREs, W. (1994): Changes in the Geo-Biosphere during the last 15000 years. Continental sediments as evidence of changing environmental conditions. IGBP Informationsbrief 16: 1-2; Berlin.

Andres, W., Litt, Th., Merkt, J., Müller-Beck, H., Schmincke, H.-U., Schneider, J. \& Welte, H. (1993): Wandel der Geo-Biosphäre während der letzten 15000 Jahre. Kontinentale Sedimente als Ausdruck sich verändernder Umweltbedingungen. Antrag auf Einrichtung eines Schwerpunktprogramms bei der DFG, 27 S., unveröffentlicht.

BANGERT, V. (1958): Über Rheinarme und Grundwasserverhältnisse südlich des Kaiserstuhls.- Ber. Naturf. Ges. Freiburg i.Br. 48: 159-166; Freiburg i.Br.

Dehn, R. (19842): Neue Ergebnisse zur urgeschichtlichen Besiedlung des Kaiserstuhls. - In: Biegel, G., Dehn, R. \& Fingerlin, G.(Hrsg.): Neue Ausgrabungen. Archäologische Denkmalpflege im Regierungsbezirk Freiburg; Freiburg i. Br. (Verlag K. Schillinger).

- (1988): Tarodunum und Kegelriß. Neues zur Spätlatènezeit im Breisgau. - Denkmalpflege in Bad.Württ. 17: 94-97.

- (1989): Zu spätlatènezeitlichen Siedlungen im Breisgau.- In: Marburger Kolloquium 1989, Wolfgang Dehn zum 80. Geburtstag. S. 89-99; Marburg.

Dieckmann, B. (1990): Die Kulturgruppen Wauwil und Strassburg im Kaiserstuhlgebiet. - Cahiers de l'Association de la promotion de la recherche archeologique en Alsace 6: 7-60; Zimmersheim.
- (1991): Zum Mittel- und Jungneolithikum im Kaiserstuhlgebiet. - Diss. Phil. Fak. Univ. Freiburg, 395 S., unveröffentlicht.

FingerLIN, G. (1991): Ur- und Frühgeschichte auf Grund der archäologischen Quellen. - Ber. Naturf. Ges. Freiburg i.Br. 81: 65-116; Freiburg i. Br.

Foellmer, A., Hoppe, A. \& Dehn, R. (1997): Anthropogene Schwermetallanreicherungen in holozänen Auensedimenten der Möhlin (südlicher Oberrheingraben). - Die Geowissenschaften 15: 61-66.

Friedmann, A. (1998): Pollenanalytische Untersuchungen im Wasenweiler Ried (Südbaden). - In: MäckeL, R. \& Friedmann, A. (Hrsg.): Wandel der Geo-Biosphäre in den letzten 15000 Jahren im südlichen Oberrheintiefland und Schwarzwald. Freiburger Geogr. Hefte 54: 163-174; Freiburg i.Br.

FriedmanN, A. \& Mäckel, R. (1998): Die Landschaftsentwicklung in den Lößgebieten des Kaiserstuhls und Tunibergs. - In: Mäckel, R. \& Friedmann, A. (Hrsg.): Wandel der Geo-Biosphäre in den letzten 15000 Jahren im südlichen Oberrheintiefland und Schwarzwald. Freiburger Geogr. Hefte 54: 99-112; Freiburg i.Br.

KAYSER, S. \& MÄCKEL, R. (1994): Fluviale Geomorphodynamik und Reliefentwicklung im Ostrheingebiet. Ber. Naturf. Ges. Freiburg i. Br. 82/83: 93-115; Freiburg i.Br.

LaIs, R. (1934): Das nördliche Kaiserstuhlvorland, seine Bodengestalt, Entstehungsgeschichte und frühe Besiedlung.- Schau-ins-Land 61: 9-20.

- (1935): Zur vor- und frühgeschichtlichen Besiedlung des nördlichen Kaiserstuhlvorlandes. - Bad. Fundber. 3/1933-36: 181-191

Lehmann-Carpzov, R., Paternoster, K. \& StubendorfF, U. (1978): Quartärgeologische Deckschichten-Kartierung im Wasenweiler Ried zwischen Kaiserstuhl und Tuniberg (Südbaden). - Jh. geol. Landesamt Bad-Württ. 20: 77-100; Freiburg i. Br.

Ludemann, T. (1996): Die Wälder im Sulzbachtal (Südwest-Schwarzwald) und ihre Nutzung durch Bergbau und Köhlerei. - Mitt. Ver. Forstl. Standortkunde u. Pflanzenzüchtung 38: 87-118.

Mäckel, R. (1997): Spät- und postglaziale Flußaktivität im Schwarzwald und Oberrheintiefland. - In: MÄCKEL, R. \& B. Metz (Hrsg.): Schwarzwald und Oberrheintiefland. Eine Einführung in das Exkursionsgebiet um Freiburg im Breisgau, 2. Auflage. Freiburger Geogr. Hefte 36: 75-99; Freiburg i. Br.

- (1998): Flußaktivität und Talgeschichte des Spätund Postglazials im Oberrheintiefland und Schwarzwald. - In: Mäckel, R. \& Friedmann, A. (Hrsg.): Wandel der Geo-Biosphäre in den letzten 15000 Jahren im südlichen Oberrheintiefland und Schwarzwald. Freiburger Geogr. Hefte 54: 31-50; Freiburg i.Br.

— \& Zoluinger, G. (1989): Fluvial action and valley development in the Central Southern Black Forest during the Late Quaternary. - In: Catena Suppl. 15: 243-252.

- \& RöHrig, A. (1991): Flußaktivität und Talentwicklung des Mittleren und Südlichen Schwarzwaldes und Oberrheintieflandes. - Ber. z. Dt. Landeskunde 65: 287-311. 
- \& Zollinger, G. (1995): Holocene river and slope dynamics in the Black Forest and Upper Rhine Lowlands under the impacts of man. - Z. Geomorph. N. F. Suppl.-Bd. 100: 89-100.

MÄHling, W. (1982): Urgeschichtliche Siedlungslandschaften in der Vorbergzone des südlichen Oberrheingebietes: Das nördliche Markgräfler Hügelland.- Das Markgräflerland 1982/1: 3-41; Schopfheim.

Rudloff, H. v. (1980): Die Klima-Entwicklung in den letzten Jahrhunderten im mitteleuropäischen Raume (mit einem Rückblick auf die postglaziale Periode).- In: Oeschger, H., Messerli, B. \& Svilar, M. (Hrsg.): Das Klima. Analysen und Modelle, Geschichte und Zukunft. S. 125-148.

Schmid, E. \& Stülpnagel, W. (1965): Ur- und Frühgeschichte. - In: Statist. Landesamt Baden-Württemberg (Hrsg.): Freiburg im Breisgau, Stadtkreis und Landkreis, Bd. I/1: 148-180; Freiburg i. Br.

Schmincke, H.-U. \& BogaARd, P. v.D. (1991): Tephra layers and tephra events. - In: Einsele, G., Ricken, W. \& SeilacheK, A. (Hrsg.): Cycles and events in stratigraphy. Heidelberg, Berlin, New York (Springer).

SchreIner, A. (1958): Niederterrasse, Flugsand und Löß am Kaiserstuhl (Südbaden). - Mitt. bad. Landesv. Naturkde. u. Natschutz. N. F. 7: 113-125; Freiburg i. $\mathrm{Br}$.

- (1959): Das Quartär. - In: GLA (Hrsg.): Erläuterungen zur geologischen Exkursionskarte des Kaiserstuhls 1:25000. S. 77-89; Stuttgart.

- (19963): Quartär. - In: GLA (Hrsg.): Erläuterungen zur geologischen Karte von Freiburg i. Br. und Umgebung. S. 174-199; Freiburg i. Br.

Sleumer, H. (1934): Eine pollenanalytische Untersuchung des Wasenweiler Riedes. - Mitt. bad. Landesver. f. Naturkde. u. Natschutz. N. F. 3: 25-28; Freiburg i.Br.

Steuer, H. (1990): Zur Frühgeschichte des Erzbergbaus und der Verhüttung im südlichen Schwarzwald.- In: Nuber, H. U., Schmid, K., Steuer, H. \& Zotz, Th. (Hrsg.): Archäologie und Geschichte des 1. Jahrtausends in Südwestdeutschland, Archäologie und Geschichte, Freiburger Forschungen zum 1. Jahrtausend in Südwestdeutschland, Band 1: 387-415; Sigmaringen.
- \& Zimmermann, U. (Hrsg., 1993a): Alter Bergbau in Deutschland. - Archäologie in Deutschland. Sonderheft 1993, 127 S.; Stuttgart (Theiss).

— \& Zimmermann, U. (Hrsg., 1993b): Montanarchäologie in Europa. Archäologie und Geschichte. - Freiburger Forschungen zum ersten Jahrtausend in Südwestdeutschland, Bd. 4; Sigmaringen.

Weber-Jenisch, G. (1995): Der Limberg bei Sasbach und die spätlatènezeitliche Besiedlung des Oberrheingebietes. - Veröff. d. Landesdenkmalamts Ba.-Wü.; Stuttgart.

Zimmermann, U. (1990): Die Ausgrabungen in alten Bergbaurevieren des südlichen Schwarzwaldes.In: Steuer, H: \& Zimmermann, U. (Hrsg.): Erze, Schlacken und Metalle. Freiburger Universitätsblätter 109: 115-146; Freiburg i. Br.

Zollinger, G. (1990): Quartäre Geomorphogenese und Substratentwicklung am Schwarzwald-Westrand zwischen Freiburg und Müllheim (Südbaden). Physiogeographica. Basler Beiträge zur Physiogeographie 12, 202 S.; Basel.

— \& Bucher, B. (1989): Erosionsmessungen im Norsinger Ahbach südlich von Freiburg und ihre geomorphologische und hydrologische Interpretation. Ber. Naturf. Ges. Freiburg i. Br. 77/78: 67-79; Freiburg i.Br.

— \& MäCKEL, R. (1989): Quartäre Geomorphodynamik im Einzugsgebiet des Sulzbaches und der Möhlin, Südbaden. - Ber. Naturf. Ges. Freiburg i. Br. 77/78 81-98; Freiburg i.Br.

Manuskript eingegangen am 26. März 1998 